\title{
Modulation of Mean Wind and Turbulence in the Atmospheric Boundary Layer by Baroclinicity $\mathcal{O}$
}

\author{
MOSTAFA MOMEN \\ Department of Civil and Environmental Engineering, Princeton University, Princeton, New Jersey, and Department of \\ Civil Engineering and Engineering Mechanics, Columbia University, New York, New York \\ ELIE BOU-ZEID \\ Department of Civil and Environmental Engineering, Princeton University, Princeton, New Jersey \\ MARC B. PARLANGE \\ Department of Civil Engineering, Monash University, Melbourne, Victoria, Australia \\ MARCO GIOMETTO \\ Department of Civil Engineering and Engineering Mechanics, Columbia University, New York, New York
}

(Manuscript received 11 June 2018, in final form 9 August 2018)

\begin{abstract}
This paper investigates the effects of baroclinic pressure gradients on mean flow and turbulence in the diabatic atmospheric boundary layer (ABL). Large-eddy simulations are conducted where the direction of the baroclinicity, its strength, and the surface buoyancy flux are systematically varied to examine their interacting effects. The thermal wind vector, which represents the vertical change in the geostrophic wind vector resulting from horizontal temperature gradients, significantly influences the velocity profiles, the Ekman turning, and the strength and location of the low-level jet (LLJ). For instance, cold advection and positive (negative) geostrophic shear increased (decreased) friction velocity and changed the LLJ elevation. Given the baroclinicity strength and direction under neutral conditions, a simple reduced model is proposed and validated here to predict the general trends of baroclinic mean winds. The baroclinic effects on turbulence intensity and structure are even more intricate, with turbulent kinetic energy (TKE) profiles displaying an increase of TKE magnitude with height for some cases. When a fixed destabilizing surface heat flux is added, a positive geostrophic shear favors streamwise aligned roll-type structures, which are typical of neutral ABLs. Conversely, a negative geostrophic shear promotes cell-type structures, which are typical of strongly unstable ABLs. Furthermore, baroclinicity increases shear in the outer ABL and tends to make the outer flow more neutral by decreasing the Richardson flux number. These findings are consequential for meteorological measurements and the wind-energy industry, among others: baroclinicity alters the mean wind profiles, the TKE, coherent structures, and the stability of the ABL, and its effects need to be considered.
\end{abstract}

\section{Introduction}

Modeling geophysical flows to accurately predict the weather and climate requires a comprehensive understanding of the physics of the atmospheric boundary

Supplemental information related to this paper is available at the Journals Online website: https://doi.org/10.1175/JAS-D-180159.s1.

Corresponding author: Mostafa Momen, mymomen@gmail.com layer (ABL). The ABL imposes the bottom boundary conditions on larger-scale atmospheric flows; it is the main source of thermal energy as well as trace gases, and the main sink of momentum and kinetic energy for the whole atmosphere. ABL flows are mainly driven by horizontal pressure gradients and buoyancy forces, with two additional important components: friction and Coriolis forces. In real-world ABLs, the pressure gradient force can vary in time (unsteadiness) and height (baroclinicity). However, the large majority of previous studies of ABL flows have focused on barotropic 
conditions, with a time-constant horizontal pressure gradient (Blackadar 1957; Deardorff 1972; Ekman 1905; Monin and Obukhov 1954; Shah and Bou-Zeid 2014). This idealization has also been the focus of most books on the subject, since it facilitated the development of a theoretical understanding of ABL behavior and turbulence closure models. Nevertheless, with the increasing need for high accuracy models for the thermo-fluid dynamics of the ABL in a very wide range of applications-including wind energy, urban meteorology, and pollutant dispersion-the need to incorporate the additional layers of complexity (that characterizes real-world conditions) becomes pressing. Some of these complexities-including heterogeneity in the surface properties (e.g., complex terrains; Chow and Street 2009), clouds and moisture flux in the ABL (Holtslag et al. 2013), capping inversion layer (Pedersen et al. 2014), and unsteadiness (e.g., Momen and Bou-Zeid 2015, 2016b, 2016a; Van de Wiel et al. 2010) - are being increasingly examined in the literature. In comparison, however, the departure from barotropic conditions has received less attention.

A barotropic environment arises when horizontal density variations are only induced by horizontal pressure variations. On the other hand, in a baroclinic environment, density varies because of both horizontal pressure and temperature changes. As a consequence, the gradients of pressure and density are not necessarily aligned under baroclinic conditions. The influence of this misalignment appears in the momentum conservation equation as a modification to the pressure gradient force term $-\rho^{-1} \nabla p$. This can alter the mean acceleration and velocity in various regions of the flow, thus affecting the turbulence structure and statistics. There are other impacts of baroclinicity on vorticity generation (Kundu and Cohen 2008), on large-scale horizontal instability in geophysical cold filaments (Gula et al. 2014), and other large-scale atmospheric instabilities (Vallis 2006, 104116, 261-263) that we do not consider in this study. For instance, baroclinicity influences the vorticity generation of flows (Kundu and Cohen 2008) that could be important for studying the dynamics of gravity currents (Cantero et al. 2008; Momen et al. 2017b). Since air density inherently depends on temperature, baroclinicity will emerge whenever temperatures in the atmosphere vary on horizontal scales commensurate with those of density and pressure. Such conditions occur quite often (Wyngaard 2010) especially at midlatitudes, for example, from strong horizontal gradients in temperature that form across fronts (Rizza et al. 2013) and across land-water or ice-water boundaries (Floors et al. 2015). However, despite observational evidence and theoretical support that point to the prevalence of baroclinic conditions during such critical meteorological events (Sheppard et al. 1952; Venkatesh and Csanady 1974; Hoxit 1974; Arya and Wyngaard 1975), there has been a remarkably limited number of studies examining the impact of such conditions on the ABL. Bridging this knowledge gap is particularly critical since baroclinic conditions might influence important processes and modulate the development of these events, and since baroclinicity is often ignored when observational data are being analyzed.

A few numerical studies have examined some aspects of the baroclinic ABLs. In particular, large-eddy simulations (LESs) were employed to investigate the mean and turbulence profiles in the ABL and determine the unique characteristics of baroclinic flows. Brown (1996) performed baroclinic LESs to evaluate the performance of two simple closures and showed that the presence of shear in the geostrophic winds does not significantly impact this performance. However, he demonstrated that with greater shear in the geostrophic winds of a hybrid baroclinic case (negative shear plus cold advection; these will be further explained later) the shear production of turbulent kinetic energy (TKE) becomes negligible at around $0.25 z_{i}\left(z_{i}\right.$ is the height of the base of the inversion layer) and the transport terms are no longer negligible in the TKE budget above this elevation. Strong baroclinicity might therefore challenge the validity of diagnostic stress-TKE relationships, or 1.5-order closure schemes, which are based on the assumption that production and dissipation are the leading-order terms in the TKE budget equation. Furthermore, Brown (1996) showed that an increase of geostrophic shear leads to significant changes in the stress and velocity profiles. Baroclinicity, in conjunction with stability and Earth's rotation, were shown to be the main governing parameters for determining the equilibrium depths of neutral and stable ABLs; Zilitinkevich and Esau (2003) presented diagnostic equations to capture these equilibrium heights and validated their results against LESs for neutral and stable ABLs.

Baroclinicity is known to modify not only wind and temperature profiles, but also the second-order turbulence statistics in the ABL (Sorbjan 2004). For instance, Sorbjan (2004) used LESs to improve the convective scaling introduced by Deardorff (1970) in unstable ABLs by recognizing the presence of two different regimes in the mixed layer. The first regime was identified in the core part of the mixed layer, which is controlled by surface heating and can be described by the convective scaling of Deardorff (1970), while the second regime characterizes the top of the mixed layer and is controlled by thermal stratification and shear. The latter does not obey 
Deardorff's scaling. Pedersen et al. (2013) also investigated the effect of unsteady and baroclinic forcing on the wind profiles with the help of LESs and compared their results with two case studies of the daytime ABL. In both cases, they found that including height and time variations in the applied pressure gradient forcing improves agreements with measured wind speeds. Previous studies also indicate that baroclinic conditions will modify entrainment at the top of the ABL (Brown 1996; Hess 2004) and the cross-isobaric and crossisothermal wind angles, thus affecting Ekman pumping and frontogenesis (Sheppard et al. 1952; Venkatesh and Csanady 1974; Arya and Wyngaard 1975), as well as the strength of low-level jets (Floors et al. 2015). These results have highlighted the differences between barotropic and baroclinic ABLs, and motivate the need for additional studies to better understand these differences and their implications for accurate modeling of realworld ABLs.

A horizontally homogeneous pressure gradient can be represented as an equivalent horizontal geostrophic wind, with components $U_{g}$ and $V_{g}$. However, while in a barotropic ABL these components are independent of height $z$, in a baroclinic ABL they can vary independently with $z$

$$
\left[U_{g}(z, t), V_{g}(z, t)\right] \equiv\left[-\frac{1}{f \rho} \frac{\partial \bar{p}}{\partial y}(z, t), \frac{1}{f \rho} \frac{\partial \bar{p}}{\partial x}(z, t)\right],
$$

where $f$ is the Coriolis parameter (here taken as $1.394 \times$ $10^{-4} \mathrm{~s}^{-1}$ at $73^{\circ} \mathrm{N}$; its absolute value is not particularly relevant since we nondimensionalize all of our results); $\rho$ is the fluid density; $p$ is the pressure; $x, y$, and $z$ are positions along the horizontal and vertical directions; and the overbar denotes Reynolds averaging (here surrogated for by space and/or time averaging as appropriate). Differentiating Eq. (1) with respect to $z$ and using the hydrostatic approximation and the ideal gas law (Brown 1996), the equations of the thermal wind, defined as the vertical gradient of the geostrophic wind, can be written in terms of the time average (equivalent to a Reynolds average here and denoted by an overbar) of the potential temperature $\theta$ :

$$
\begin{aligned}
& \frac{\partial U_{g}}{\partial z}=-\frac{g}{f \theta_{r}} \frac{\partial \bar{\theta}}{\partial y}+\frac{U_{g}}{\theta_{r}} \frac{\partial \bar{\theta}}{\partial z}, \\
& \frac{\partial V_{g}}{\partial z}=+\frac{g}{f \theta_{r}} \frac{\partial \bar{\theta}}{\partial x}+\frac{V_{g}}{\theta_{r}} \frac{\partial \bar{\theta}}{\partial z},
\end{aligned}
$$

where $\theta_{r}(\mathrm{~K})$ is the Boussinesq reference potential temperature, corresponding to the reference density $\rho_{r}\left(\mathrm{~kg} \mathrm{~m}^{-3}\right)$. The last terms on the right-hand side of the above equations are negligible if the vertical temperature gradient is close to the adiabatic lapse rate (Arya and Wyngaard 1975). Therefore, the dominant terms in the geostrophic shear are typically the horizontal temperature gradients.

This phenomenon has led many researchers (e.g., Hess 1973; Arya and Wyngaard 1975), to develop theoretical frameworks and numerical models using baroclinicity parameters based on the horizontal temperature gradients in Eqs. (2) such as

$$
M_{x, 0}=\frac{z_{i}}{u_{*}}\left(\frac{\partial U_{g}}{\partial z}\right)_{0}, \quad M_{y, 0}=\frac{z_{i}}{u_{*}}\left(\frac{\partial V_{g}}{\partial z}\right)_{0},
$$

where $u_{*}$ is the friction velocity at the surface, $z_{i}$ is the ABL depth, and subscript 0 refers to quantities evaluated at $z=z_{0}$ (the aerodynamic roughness length). Equivalently, these parameters could be presented as the magnitude of the vector $M_{0}=\sqrt{M_{x, 0}^{2}+M_{y, 0}^{2}}$ and its orientation angle $\beta=\tan ^{-1}\left(M_{y, 0} / M_{x, 0}\right)$. Similarity or approximate analytical models (based on the solution of the Ekman equations with height-varying geostrophic wind) that account for these two parameters have been tested and have shown improved skill in matching observational case studies (Venkatesh and Csanady 1974; Arya and Wyngaard 1975; Berger and Grisogono 1998; Tan 2001; Hess 2004; Djolov et al. 2004; Nieuwstadt 1983) or have even explained observations that simply cannot exist in a barotropic $\mathrm{ABL}$ such as wind blowing toward higher pressure (Sun et al. 2013).

Nevertheless, the emergence of two new parameters is a major barrier to the comprehensive understanding of baroclinic ABLs. Because of constraints in real-world observations, field experimental studies have only investigated a limited range of baroclinic regimes, without offering a full picture that can lead to a comprehensive understanding. Simulation efforts, particularly those focusing on idealized LESs that systematically vary $M_{x, 0}$ and $M_{y, 0}$, are well suited to provide insight. However, there have only been a few such studies (e.g., Brown 1996; Sorbjan 2004; Brown et al. 2006) that systematically probed the parameter space of this problem and the effects on mean and turbulence behavior, compared to tens of studies on barotropic ABLs conducted each year. Other efforts have applied LESs to specific barotropic/baroclinic observational periods [e.g., LESs of diurnal cycle with varying geostrophic forcing; Kumar et al. (2010); Basu et al. (2008); Pedersen et al. (2013)]. These studies could not use the directly measured pressure gradient field or large-scale horizontal temperature gradient to obtain the real-world geostrophic wind profiles, mainly because of limitations in measurement data, which resulted in some uncertainty. 
They had to resort to mesoscale simulations (or strong assumptions about the baroclinicity in the measured data) to deduce these parameters. More importantly, they did not aim to develop comprehensive theories for the baroclinic ABL by investigating the full parameter space. It is important to note that these efforts, despite their pioneering significance, were not able to fully probe the interaction of baroclinicity and various stability conditions (e.g., these studies did not examine the stable baroclinic ABL). This is, in part, because baroclinicity adds two new dimensionless parameters [Eq. (3)] to the problem compared to barotropic conditions, making the flow system more challenging to characterize. Another reason for overlooking stable stratifications in earlier studies might be related to the higher resolution required in such simulations. In summary, important aspects of the problem, such as the influence of baroclinicity on the Ekman turning, the low-level jet, TKE budget profiles, and large-scale turbulence structures, are yet to be probed.

This gap in knowledge motivates the present study where we investigate the baroclinic ABL by performing a suite of LESs including stability effects. In particular, we intend to address the following questions:

1) What are the most significant changes in the $A B L$ mean wind and turbulence characteristics resulting from baroclinicity?

2) How do $M_{0}$ and $\beta$ jointly regulate the effects of the baroclinicity, and what are the distinctions between weakly and strongly baroclinic ABLs?

3) How does static stability modulate baroclinic effects on the ABL?

The LES technique and numerical details of the code are described in the next section. In section 3, we examine the impacts of baroclinicity on the mean and turbulence characteristics of neutral ABLs. Then, we develop an analytical model for neutral baroclinic ABLs that predicts the velocity profiles, given baroclinicity strength and direction, and validate its solutions against LES results for five different baroclinic scenarios and a barotropic case. In section 4, we continue our analysis by examining the interacting effects of baroclinicity and stability on the mean and turbulence behavior. A summary and conclusions follow in section 5 .

\section{Details of the numerical simulations}

\section{a. Governing equations}

We use the LES technique, which is well established by now, and thus we will only detail the aspects of the numerical simulations that are unique to the baroclinic ABL. Since our LES code [detailed in Bou-Zeid et al. (2005)] uses pseudospectral numerical schemes in the horizontal directions, with embedded periodic boundary conditions, some modifications to the equations are required to avoid solving for the resolved potential temperature $\theta$, the mean of which will be spatially variable in the horizontal directions and hence nonperiodic under baroclinic conditions. In line with Sorbjan (2004), we first define the baroclinic horizontally varying, time-averaged mean field $\Theta(x, y, z)$ that can be assumed to have constant horizontal gradients $\partial \Theta / \partial x$ and $\partial \Theta / \partial y$ (horizontal temperature variability scale much greater than turbulent eddy scale). Then we define its horizontal mean $\langle\Theta\rangle_{p}(x-y$ planar averaging is denoted by angle brackets with subscript $p$ ) that varies in $z$, and the corresponding spatial perturbation $\Theta^{\prime \prime}=\Theta-\langle\Theta\rangle_{p} ;$ note that $\partial \Theta / \partial x=\partial \Theta^{\prime \prime} / \partial x$ and $\partial \Theta / \partial y=$ $\partial \Theta^{\prime \prime} / \partial y$. We can now define the modified turbulent instantaneous temperature $\theta^{*}=\theta-\Theta^{\prime \prime}=\theta+\langle\Theta\rangle_{p}-\Theta$, which will be horizontally periodic. By time averaging the previous relation we obtain $\left\langle\theta^{*}\right\rangle_{t}=\langle\Theta\rangle_{p}$ (time averaging is denoted by angle brackets with subscript $t$ ). The budget equation of $\theta^{*}$ can then be easily derived from that of $\theta$ [see Eq. (7) below] after noting that the advective terms are related by

$$
\begin{aligned}
u_{i} \frac{\partial \theta}{\partial x_{i}} & =u_{i} \frac{\partial \theta^{*}}{\partial x_{i}}+u_{i} \frac{\partial \Theta^{\prime \prime}}{\partial x_{i}}=u_{i} \frac{\partial \theta^{*}}{\partial x_{i}}+u \frac{\partial \Theta^{\prime \prime}}{\partial x}+v \frac{\partial \Theta^{\prime \prime}}{\partial y}+w \frac{\partial \Theta^{\prime \prime}}{\partial z} \\
& =u_{i} \frac{\partial \theta^{*}}{\partial x_{i}}+u \frac{\partial \Theta}{\partial x}+v \frac{\partial \Theta}{\partial y}
\end{aligned}
$$

where $x_{i}$ is the position vector and $u_{i}=(u, v, w)=$ $\left(u_{1}, u_{2}, u_{3}\right)$ is the velocity vector. Here, we have assumed that $\partial \Theta^{\prime \prime} / \partial z=0$ (i.e., the spatial perturbations of the time-averaged baroclinic temperatures are constant with height), which is equivalent to assuming that the horizontal temperature gradient is height invariant. We note again that $\theta^{*}$ is periodic and can thus be solved for numerically using our pseudospectral code.

The buoyancy force appearing in the momentum equation will now be proportional to the potential temperature perturbation from the mean of the isothermal (of constant potential temperature) surface since this is the surface of neutral buoyancy for the parcel. In barotropic environments, this is taken as the mean over a horizontal plane; however, that cannot be applied to a baroclinic environment. The perturbation from the mean over the isothermal surface on which the parcel lies (i.e., from $\Theta$ ) is needed. The buoyancy perturbation 
temperature is therefore $\theta^{\prime}=\theta-\Theta=\theta^{*}-\langle\Theta\rangle_{p}$, but since $\left\langle\theta^{*}\right\rangle_{t}=\langle\Theta\rangle_{p}$, then $\theta^{\prime}=\theta^{*}-\left\langle\theta^{*}\right\rangle_{t} \equiv \theta^{* \prime}$.

At this stage, the modified governing equations of the LES can be written for the filtered (denoted by a tilde) quantities as the continuity equation:

$$
\frac{\partial \tilde{u}_{i}}{\partial x_{i}}=0
$$

the momentum-conservation equation in rotational form:

$$
\begin{aligned}
\frac{\partial \tilde{u}_{i}}{\partial t}+\tilde{u}_{j}\left(\frac{\partial \tilde{u}_{i}}{\partial x_{j}}-\frac{\partial \tilde{u}_{j}}{\partial x_{i}}\right)= & -\frac{1}{\rho} \frac{\partial \tilde{p}^{*}}{\partial x_{i}}-\frac{\partial \tau_{i j}}{\partial x_{j}}+g \frac{\widetilde{\theta^{*}}}{\theta_{r}^{\prime}} \delta_{i 3} \\
& +f\left(U_{g}-\tilde{u}_{1}\right) \delta_{i 2}-f\left(V_{g}-\tilde{u}_{2}\right) \delta_{i 1}
\end{aligned}
$$

and the thermal energy conservation for potential temperature perturbation $\theta^{*}$ :

$$
\frac{\partial \widetilde{\theta^{*}}}{\partial t}+\tilde{u}_{j} \frac{\partial \widetilde{\theta^{*}}}{\partial x_{j}}+\tilde{u} \frac{\partial \Theta}{\partial x}+\tilde{v} \frac{\partial \Theta}{\partial y}=-\frac{\partial \pi_{j}}{\partial x_{j}},
$$

where $\tau_{i j}=\sigma_{i j}-1 / 3 \sigma_{k k} \delta_{i j}$ is the deviatoric part of the subgrid-scale (SGS) stress tensor $\sigma_{i j}=\widetilde{u_{i} u_{j}}-\tilde{u}_{i} \tilde{u}_{j}, p^{*}$ is a modified pressure defined as $\tilde{p}^{*} \equiv \tilde{p}+1 / 2 \rho \tilde{u}_{j}^{2}+1 / 3 \rho \sigma_{k k}$, $\theta_{r}$ is the reference temperature (the same for $\theta^{*}$ and $\theta$ ), $g$ is the gravitational constant, and $\pi_{j}=\widetilde{\theta^{*} u_{j}}-\widetilde{\theta^{*} \tilde{u}_{j}}$ is the SGS heat flux vector. Both the SGS deviatoric stress and the SGS heat flux are modeled using an eddy diffusivity parameterization; specifically, a dynamic Lagrangian scale-dependent Smagorinsky model is used to determine the SGS eddy viscosity $\nu_{T}$ (Bou-Zeid et al. 2005) and model $\tau_{i j}$. A constant SGS Prandtl number, $\operatorname{Pr}_{\mathrm{sgs}}=$ 0.4 , is prescribed to model $\pi_{j}=-\left(\nu_{T} / \mathrm{Pr}_{\mathrm{sgs}}\right)\left(\partial \widetilde{\theta}^{*} / \partial x_{j}\right)$ (Kumar et al. 2010). The LES equations are solved in nondimensional form, using a priori known parameters such as $z_{i}$, and $G_{0} \equiv\left(U_{g, 0}^{2}+V_{g, 0}^{2}\right)^{1 / 2}$, the magnitude of the geostrophic wind at the surface (a reference value of $8 \mathrm{~m} \mathrm{~s}^{-1}$ is taken for $G_{0}$ here). For consistency, we use a fixed value of $z_{i}=800 \mathrm{~m}$ in stable cases and $z_{i}=$ $1000 \mathrm{~m}$ in other simulations (representing the base of the capping inversion layer that we impose) as a fixed length scale for nondimensionalizing the heights in all plots to facilitate the comparison of cases in a dimensional framework. However, the effective ABL depth might be lower than $z_{i}$, particularly under stable conditions and when there is a negative geostrophic shear in neutral cases; we will use this effective height for some cases in the reduced-order model and evaluate its performance, particularly under stable conditions. Hence, the results reported in the paper are all normalized with these fixed scales. Further details on the LES model, the SGS model, and the diabatic simulation setup can be found in Albertson and Parlange (1999), Bou-Zeid et al. (2005), and Momen and BouZeid $(2017 \mathrm{a}, \mathrm{b})$. The approach used for initializing the code, the domain size, and the boundary conditions are described in appendix A. We note that the results of the code have previously been validated for many applications, for example, for barotropic neutral, unstable, and stable ABLs (Bou-Zeid et al. 2005; Kumar et al. 2010; Huang and Bou-Zeid 2013), and the new minor changes in the forcing and the temperature equation should not impact the skill of the code in numerically integrating the equations. To have a consistent comparison with observations, inputs such as the surface heat flux over $\sim 100 \mathrm{~km}^{2}$, the mesoscale horizontal temperature gradients $(\sim 100 \mathrm{~km})$, and geostrophic wind or horizontal pressure gradient data are needed, but these are not typically provided all together.

\section{b. Baroclinicity parameters}

Following the approach of Arya and Wyngaard (1975), we define two baroclinicity parameters based on the magnitude of the two normalized components of the thermal wind vector at the surface (strength parameter) and its orientation angle. However, we rescale the velocities by $G_{0}$ instead of $u_{*}$, since this is consistent with the normalization of our LES code equations and since we are more interested in outer layer profiles (see section 3a), yielding

$$
\begin{aligned}
S_{0} & \equiv \frac{z_{i}}{G_{0}} \sqrt{\left(\frac{\partial U_{g}}{\partial z}\right)_{0}^{2}+\left(\frac{\partial V_{g}}{\partial z}\right)_{0}^{2}} \\
\beta & \equiv \tan ^{-1}\left[\left(\frac{\partial V_{g}}{\partial z}\right)_{0} /\left(\frac{\partial U_{g}}{\partial z}\right)_{0}\right] .
\end{aligned}
$$

Note that $S_{0}$ and $\beta$ are here expressed in terms of surface parameters. In line with previous studies (Brown 1996; Sorbjan 2004), we assume the second vertical derivative of the geostrophic wind to be zero. This is again equivalent to assuming that the horizontal temperature gradient is height invariant [see Eq. (2)] and results in $S(z)=S_{0}$ for any $z$. The subscripts denoting surface values of the gradients are nevertheless included here for clarity.

\section{c. Suite of large-eddy simulations}

Table 1 lists the simulations conducted in the paper for various values of $S_{0}, \beta$, and surface buoyancy flux. Note that based on the definition of geostrophic velocity, Eq. (1), it is always perpendicular to the horizontal 
TABLE 1. Details of the large-eddy simulations: 23 cases with 2 baroclinicity strengths, 5 baroclinicity directions, and under 3 stabilities. The case names are constructed as follows: the first letter denotes the surface heat flux ( $\mathrm{N}$ for neutral, $\mathrm{U}$ for unstable, and $\mathrm{S}$ for stable, replaced with placeholder * in the table), the second letter represents the strength of baroclinicity (W for weak and S for strong), and the last number shows the baroclinicity direction. In the second column, the values in parentheses are the equivalent horizontal temperature gradient $\left[\mathrm{K}(100 \mathrm{~km})^{-1}\right]$. A nondimensional stability criterion, $z_{i} / L$, is reported for all cases in Table 2 .

\begin{tabular}{|c|c|c|c|c|c|}
\hline \multirow[b]{2}{*}{ Simulation name } & \multirow[b]{2}{*}{$S_{0}\left[|\Delta \theta| / \Delta x ; \mathrm{K}(100 \mathrm{~km})^{-1}\right]$} & \multirow[b]{2}{*}{$\beta$} & \multicolumn{3}{|c|}{ Heat flux $\left(\mathrm{W} \mathrm{m}^{-2}\right)$} \\
\hline & & & Stable (S) & Neutral (N) & Unstable (U) \\
\hline Barotropic $(* \mathrm{~B})$ & $0(0)$ & - & -20 & 0 & 200 \\
\hline Weak baroclinicity + positive shear $(*$ W0 $)$ & $0.25(0.85)$ & 0 & & 0 & \\
\hline Weak baroclinicity + cold advection $(*$ W90) & $0.25(0.85)$ & 90 & & 0 & \\
\hline Weak baroclinicity + hybrid case $(*$ W135) & $0.25(0.85)$ & 135 & & 0 & \\
\hline Weak baroclinicity + negative shear $(*$ W180) & $0.25(0.85)$ & 180 & & 0 & \\
\hline Weak baroclinicity + warm advection $(*$ W270) & $0.25(0.85)$ & 270 & & 0 & \\
\hline Strong baroclinicity + positive shear $(* \mathrm{~S} 0)$ & $1(3.4)$ & 0 & -20 & 0 & 200 \\
\hline Strong baroclinicity + cold advection $(*$ S 90$)$ & $1(3.4)$ & 90 & -20 & 0 & 200 \\
\hline Strong baroclinicity + hybrid case $(*$ S135) & $1(3.4)$ & 135 & -20 & 0 & 200 \\
\hline Strong baroclinicity + negative shear $(*$ S180) & $1(3.4)$ & 180 & -20 & 0 & 200 \\
\hline Strong baroclinicity + warm advection $(*$ S270) & $1(3.4)$ & 270 & -20 & 0 & 200 \\
\hline
\end{tabular}

pressure gradient and under barotropic conditions the horizontal pressure and density gradients are parallel to each other: $\nabla \rho \| \nabla P \perp \mathbf{G}$. If the horizontal temperature gradient happens to be aligned with the surface pressure gradient $\left(\nabla T \| \nabla P_{0} \perp \mathbf{G}_{0}\right.$; sometimes referred to as equivalent barotropic), the effect of $\nabla T$ is simply to increase (positive shear) or decrease (negative shear) G with height, but does not cause directional shear. In such cases, $\mathbf{G}$ is parallel to the isotherms and causes no heat advection (there could still be heat advection by other processes such as ageostrophic winds). On the other hand, if $\nabla T \perp \nabla P_{0}$, then $\mathbf{G}_{0} \| \nabla T$ (note that $\nabla P \perp \mathbf{G}$, i.e., geostrophic wind is parallel to the isobars, is true at all heights) and $\mathbf{G}_{0}$ is thus perfectly normal to the isotherms, resulting in a highly effective scalar advection by the surface geostrophic wind (cold advection if $\mathbf{G}_{0}$ and $\nabla T$ point in the same direction or warm advection if they point in opposite directions). In the cold and warm advection cases, $V_{g}$ is increased and decreased, respectively, while $U_{g}$ remains constant. However, since there are no physical constraints on the relative orientation between $\nabla T$ and $\nabla P_{0}$ (e.g., see Fig. 1c), most real cases are expected to be hybrids of these four idealized scenarios (positive and negative geostrophic shear and cold and warm advection). A schematic illustration of the explanation above is featured in Fig. 1.

Such baroclinic cases can often occur in nature. For instance, Fig. 1c depicts a schematic of cold-air and warm-air advection systems in the United States that would indicate baroclinicity. The wind flows parallel to isobars and leads to advection of cold air toward the Great Plains and Gulf Coast (cold advection). Warm air is also pushed toward the northeastern United States. The thermal boundary between the cold and warm air (blue line) forms a cold front (boundary moving toward the warm side) and similarly a warm front (boundary moving toward cold side) is found in New England. Then, a low pressure (storm) center is formed where warm and cold fronts meet. Baroclinicity is also present in coastal regions.

Averaging in time and over $x-y$ planes (the latter operation should only be applied to the temperature perturbation $\theta^{*}$ and not to the original temperature $\theta$ ) is used as a surrogate for Reynolds averaging; temporal averaging is done over the last $\tau_{\mathrm{ABL}}$ to get the converged statistics, corresponding to an inertial period of the flow. The inertial time scale of ABLs is often characterized by the Coriolis frequency (Tennekes and Lumley 1972, $43-44)$ as $\tau_{\mathrm{ABL}} \equiv 2 \pi / f(\approx 12.5 \mathrm{~h}$ here $)$.

To ensure that our baroclinic numerical setup is implemented correctly, we compared our simulations with other available numerical results as a check on the consistency of the simulations and to ascertain that our findings can be compared to those other studies. We note that these numerical results are not necessarily correct (refer to discussion on validation above), but we do use these comparisons for consistency checks and the good match does support the validity of the simulation accuracy. In appendix B, we show that our wind-velocity profiles compare favorably with the positive-shear and hybrid cases of Brown (1996), where a similar SGS model, forcing, and domain size were used.

Although we incorporate the horizontal temperature advection terms in the thermal energy in Eq. (7), we observed that their effects are small in our domain (see the supplemental material). As Brown (1996) also notes, the advective heat flux associated with horizontal temperature gradients is negligible compared to the 


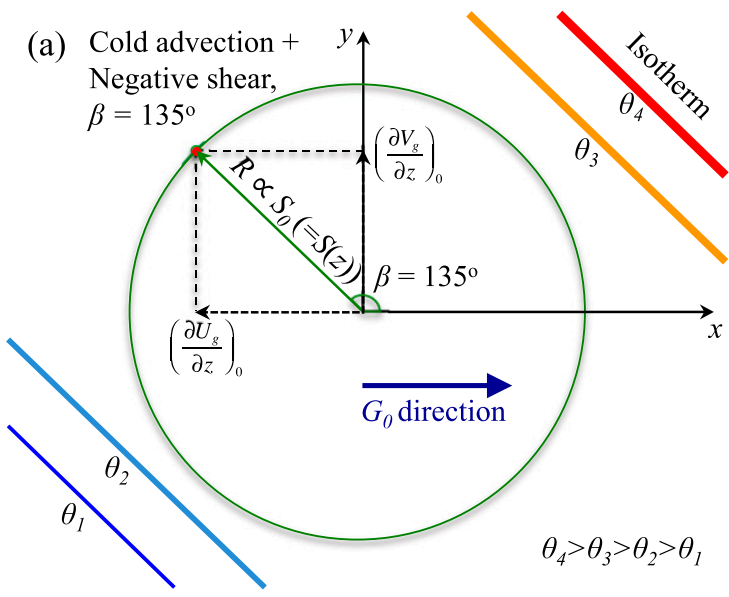

(b)
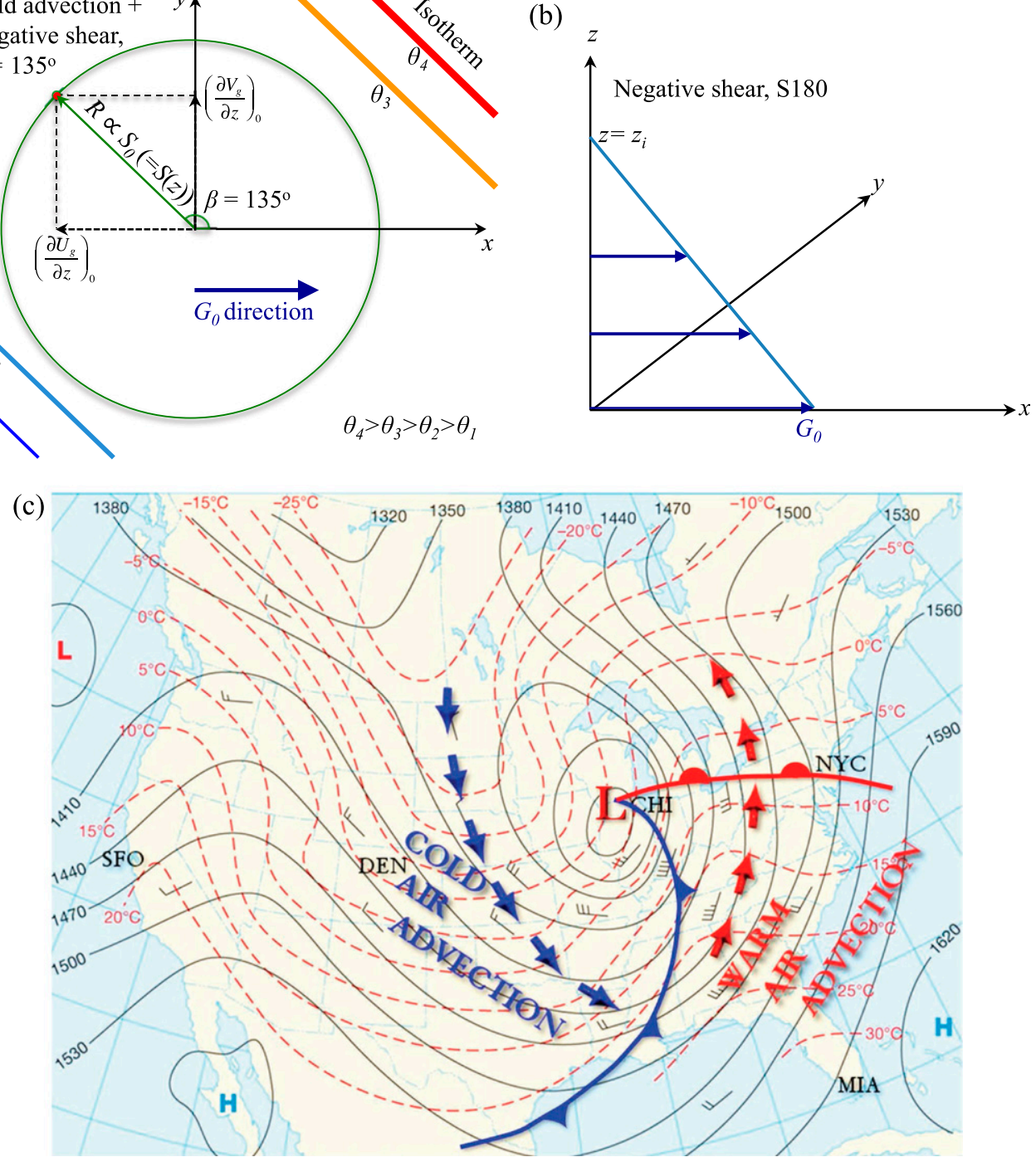

FIG. 1. Schematic view of the two parameters of the ABL for the hybrid cases (NW135, SS135, NS135, and US135), where $\beta=135^{\circ}$. Note that $\mathbf{G}_{0}$ is the geostrophic wind vector at the surface, which is along the $x$ direction here. (a) The $x-y$ plane view where the radius of the plotted circle shows the strength of the baroclinicity $S_{0}=S(z)$, which controls the magnitude of gradient of the geostrophic wind vector, while the angle $\beta$ shows the relative orientation of the surface geostrophic wind and its gradient (the thermal wind, always parallel to the isotherms). (b) The $x-y-z$ view depicting the profiles of $U_{g}(z)$ and $V_{g}(z)$ shown by the blue arrows at several heights. (c) An example of cold-air and warm-air advection cases in the United States. Solid black lines show the isopleths of equal 850-hPa height and the red dashed lines indicate isotherms (courtesy of https://www.wunderground.com/).

total heat flux (advective plus surface), and under diabatic conditions does not have a significant effect on the flow. As shown in Table 1, the horizontal temperature gradient is on the order of $10^{-5} \mathrm{~K} \mathrm{~m}^{-1}$.

\section{The effects of baroclinicity on the neutral ABL}

In this section, we examine the influence of baroclinicity strength $S_{0}$ and direction $\beta$ on the mean and turbulence characteristics of the ABL under neutral conditions.

\section{a. Implications for the mean velocity}

The ABL adapts itself to the changes in the imposed pressure gradients with height. Figure 2 shows these effects for all the neutral baroclinic cases versus a corresponding barotropic simulation. As this figure indicates, not only the strength of the baroclinicity, but also its direction can significantly change the ABL mean profiles compared to their reference barotropic counterparts (Fig. 2f). Some of the resulting wind 


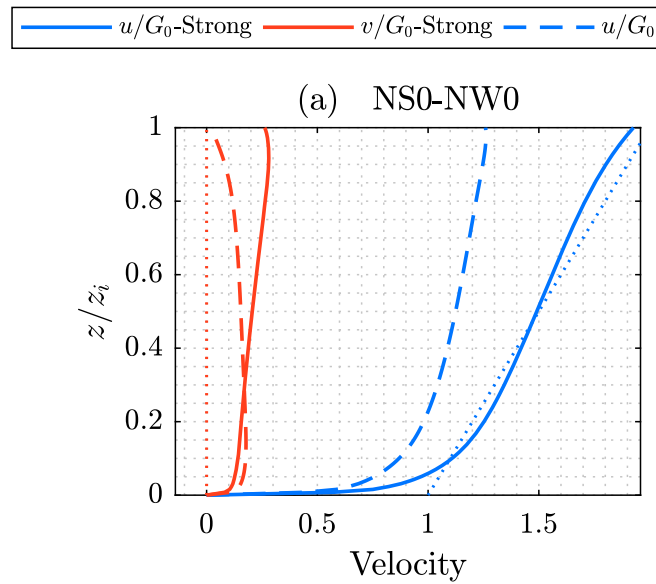

(c) NS135-NW135
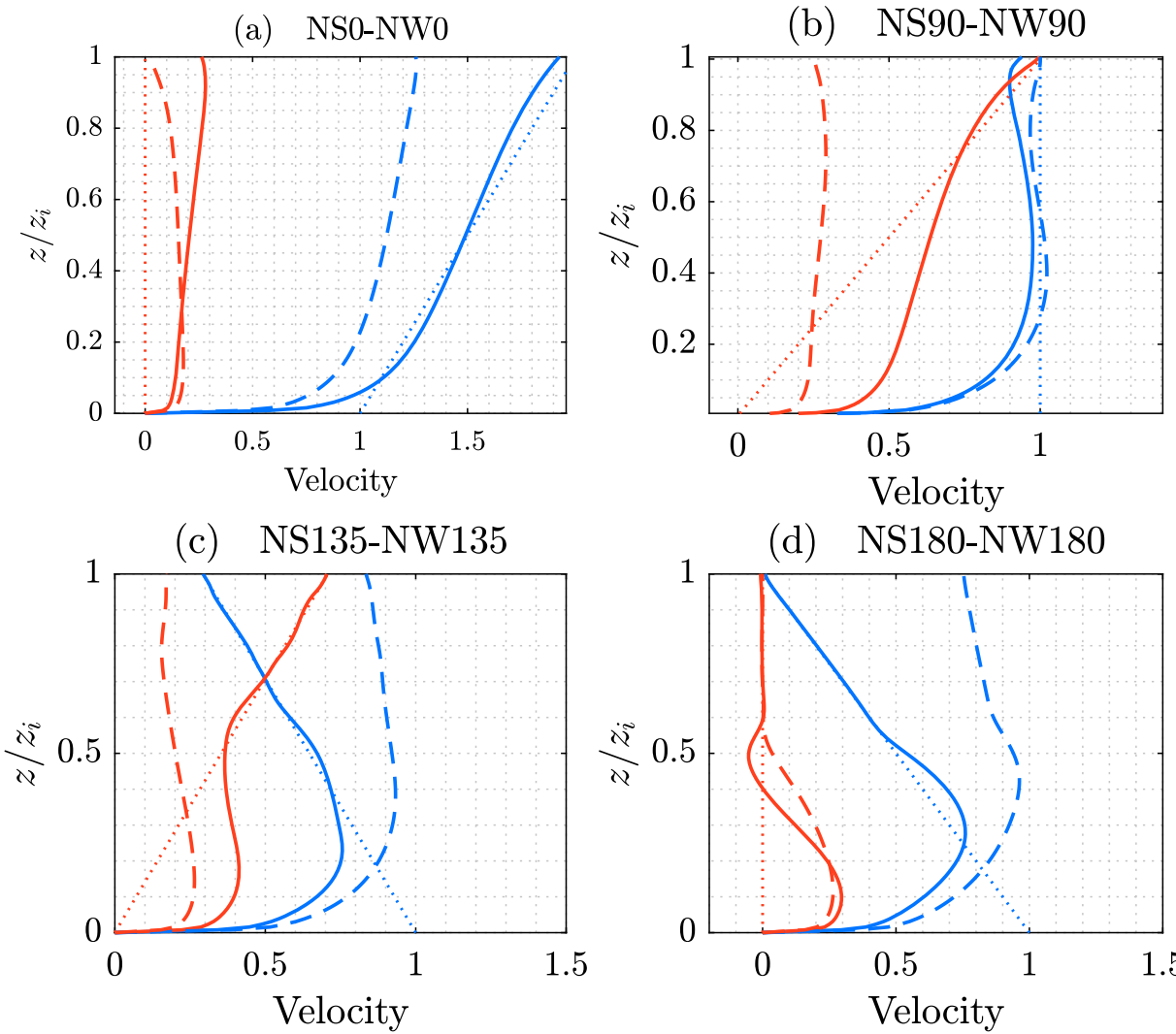

$V_{g}(z) / G_{0}$

(e) NS270-NW270

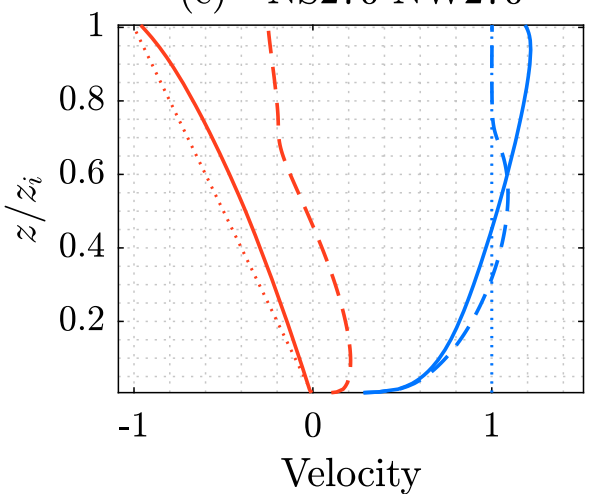

(d) NS180-NW180

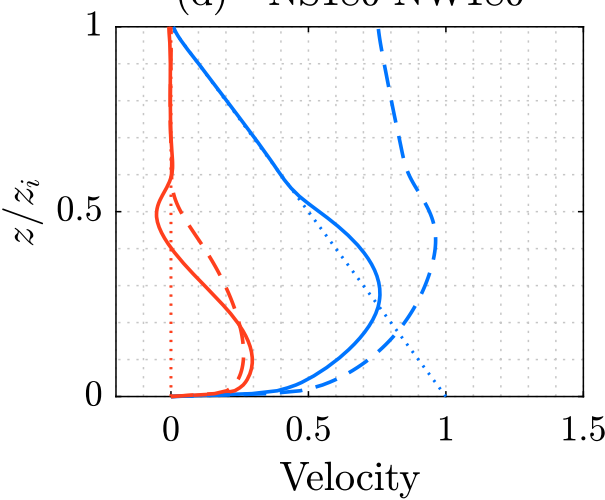

(f) NB

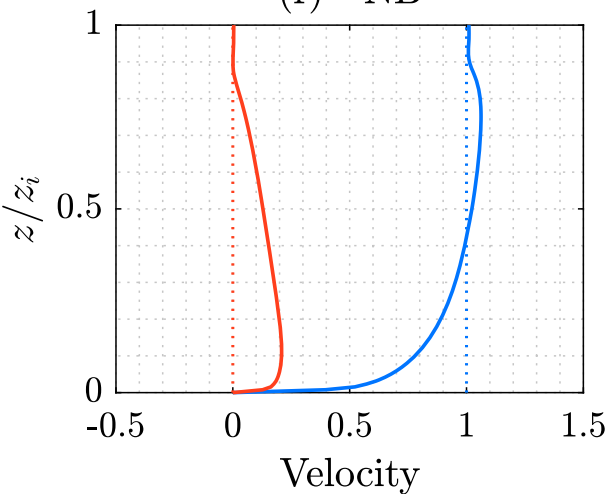

FIG. 2. Velocity profiles in strong (solid lines) and weak (dashed lines) neutral baroclinic ABLs. The dotted lines represent the geostrophic-wind profiles for the strong baroclinic cases, the forcings for the weak simulations can be inferred by dividing the slopes of the strong cases by 4 .

profiles have trends that were rarely examined. For example, the presence of a negative shear in the geostrophic wind $\left(\beta=135^{\circ}\right.$ and $180^{\circ}$, in Fig. $2 \mathrm{c}$ and Fig. 2d, respectively) results in jetlike velocity profiles that can decrease with height (to be discussed further in section 4a). In general, the velocity vectors in the ABL tend to the magnitude and direction of the geostrophic wind vectors, especially away from the surface where the turbulent friction forces induced by the surface are reduced.

Baroclinicity also strongly influences the Ekman turning. The hodographs of the mean velocities of all neutral cases are plotted in Fig. 3. Figure $3 f$ exhibits the muchstudied barotropic ABL (the classic Ekman spiral). Contrasting the barotropic hodograph (gray line in each panel) with the baroclinic ones highlights how profoundly 


- $\vec{G}(0) / G_{0} \longrightarrow$ Strong Baroclinicity - - - Weak Baroclinicity $\cdots \cdots \cdots \cdot \vec{G}(z) / G_{0}-$ NB

(a) NS0-NW0

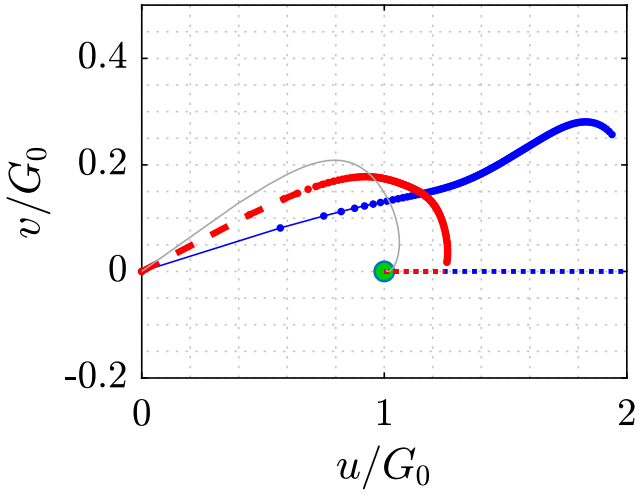

(c) NS135-NW135

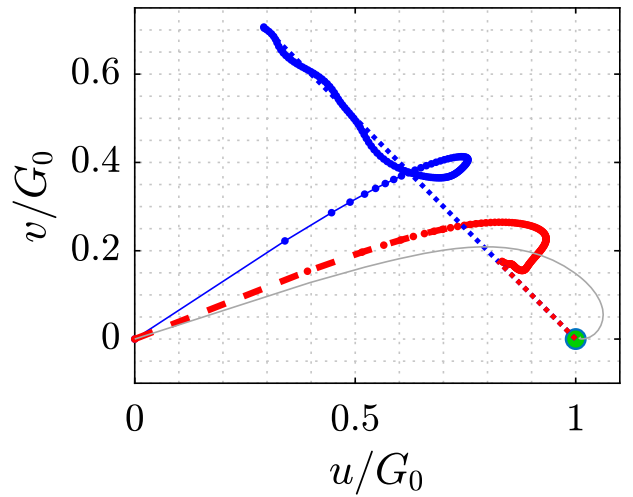

(e) NS270-NW270

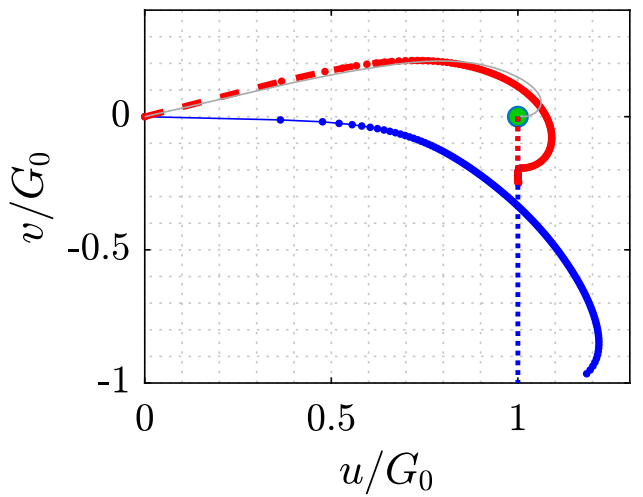

(b) NS90-NW90

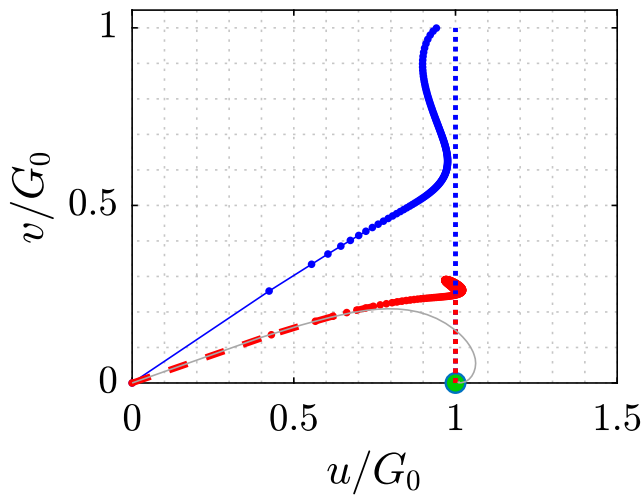

(d) NS180-NW180

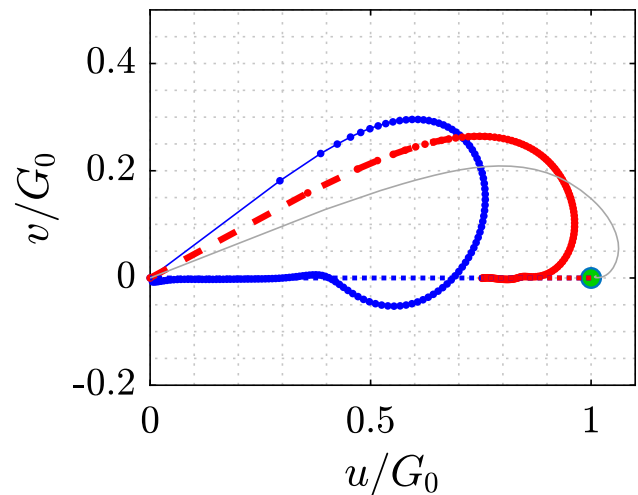

(f) $\mathrm{NB}$

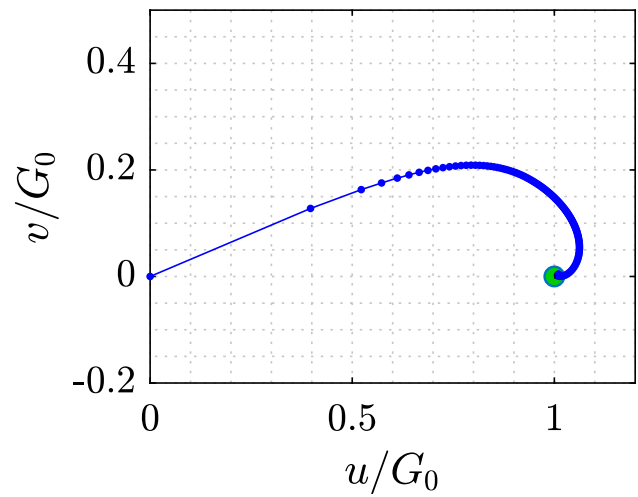

FIG. 3. The effect of baroclinicity on the velocity hodographs for all neutral cases. The velocity vectors are normalized by the magnitude of the geostrophic wind at the surface $G_{0}$. The blue and red dotted lines depict the geostrophic-wind vectors for the strong and weak baroclinicity, respectively. The points with $(u, v)=(0,0)$ denote the surface $z=z_{0}$ for the velocity plots, while the green dots represent the surface value $\mathbf{G}(0) / G_{0}=(1,0)$ of the geostrophic wind vector $\mathbf{G}(z)$, which is plotted as dashed lines.

baroclinicity impacts the mean velocity field. The hodographs are closer to the barotropic case near the surface, as could have been anticipated given that the dominant term in the near-surface region is the turbulent stress divergence and pressure effects play a smaller role. In addition, the imposed pressure gradients are closer to the barotropic value near the wall, whereas they depart more significantly from their barotropic reference away from the surface. More importantly perhaps, baroclinicity can result in a significant increase in the 
$v$ component (up to 4 times larger than the barotropic reference), which at the surface crosses the isobars in our considered cases. This implies a similar impact on Ekman pumping and significant implications at larger-meso- to synoptic - scales (see section 4 a for more details). Even if the velocities are normalized by the geostrophic wind magnitude at higher levels $G(z)$, the differences between the barotropic and baroclinic cases remain substantial. [This is illustrated in a graph in the supplemental material similar to Fig. 3, but where we normalize wind velocities with their corresponding geostrophic wind at each height, $G(z)$, instead of $G_{0}$.]

We note that an alternative velocity scale that could be used for normalization is the friction velocity $u_{*}$. This would result in a better collapse of profiles in the surface layer (i.e., $z / z_{i} \lesssim 0.2$ ) (an example is shown in the supplemental material). However, it will lead to larger discrepancies between cases in the regions away from the wall, which are the main focus of this paper. On the other hand, measuring the real-world $G(z)$ is typically more difficult than $G_{0}$, and normalizing by $G(z)$ impacts the profile shapes, thus making profiles more difficult to compare with other papers in the literature. Hence, we primarily use $G_{0}$ to normalize our results in the paper.

\section{b. Assessment of a simple model for baroclinic mean winds}

The development and validation of simple theoretical models describing the vertical structure of the mean wind in barotropic ABLs have been a main focus of ABL research. Most such models (Mahrt and Schwerdtfeger 1970; Grisogono 1995) are based on a priori prescribed, spatially variable, eddy viscosity profiles, which allow for an analytical treatment. The predictive capability of many of these models has not been evaluated for the full parameter space of baroclinic ABLs. In this section, the skills of a simple one-dimensional eddy viscosity model are quantified via comparison with LES results for the neutrally stratified baroclinic ABL. Applying the time and horizontal spatial averaging operation to Eq. (6), and modeling turbulent fluxes via $K$ theory (Stull 1988), we obtain the following equations for the steady-state, neutrally stratified, baroclinic ABL:

$$
\begin{aligned}
& \frac{d}{d z}\left(\nu_{T}^{\mathrm{tot}} \frac{d \bar{u}}{d z}\right)-f\left[V_{g, 0}+\int_{z_{0}}^{z} \frac{\partial V_{g}(z)}{\partial z} d z-\bar{v}\right]=0, \\
& \frac{d}{d z}\left(\nu_{T}^{\text {tot }} \frac{d \bar{v}}{d z}\right)+f\left[U_{g, 0}+\int_{z_{0}}^{z} \frac{\partial U_{g}(z)}{\partial z} d z-\bar{u}\right]=0 .
\end{aligned}
$$

where the vertical gradients of geostrophic winds in our considered cases can be represented in terms of $S_{0}, U_{g, 0}, V_{g, 0}$, and $\beta$ as $\partial U_{g} / \partial z=S_{0} \sqrt{U_{g, 0}^{2}+V_{g, 0}^{2}}\left(z_{i} \sqrt{1+\tan ^{2} \beta}\right)^{-1}$ and $\partial V_{g} / \partial z=S_{0} \tan \beta \sqrt{U_{g, 0}^{2}+V_{g, 0}^{2}}\left(z_{i} \sqrt{1+\tan ^{2} \beta}\right)^{-1}$. The terms $U_{g, 0}, V_{g, 0}, S_{0}$, and $\beta$ are prescribed according to Table 1, to allow for a proper comparison with the corresponding LES cases. Equations (9) are a system of secondorder ordinary differential equations (ODEs) for the $u$ and $v$ velocities, functions of $z$ in the interval $\left[z_{0}, L_{z}\right]$, where $L_{z}$ is the height of the domain (see appendix A). In Eqs. (9) $\nu_{T}^{\text {tot }}\left(\mathrm{m}^{2} \mathrm{~s}^{-1}\right)$ is the total kinematic eddy viscosity, a heightdependent, dimensional model parameter that needs to be prescribed to account for the vertical momentum diffusion due to turbulent motions. The first term in the equation represents the turbulent stress, the second term is the surface level pressure gradient forcing, and the third is the change in pressure gradient forcing with height, while the last term represents the Coriolis force. A similar eddy viscosity to O'Brien's profile (O'Brien 1970), which was proposed for use in models of planetary boundary layers in both barotropic and baroclinic situations (e.g., Nieuwstadt 1983), is adopted here as follows:

$$
\begin{aligned}
& \nu_{T}^{\text {tot }}=u_{*} \kappa z\left(1-\frac{z}{z_{i}}\right)^{2}+\varepsilon, \quad z_{0} \leq z \leq z_{i}, \\
& \nu_{T}^{\text {tot }}=\varepsilon, \quad z_{i}<z \leq L_{z},
\end{aligned}
$$

where $\kappa$ is the von Kármán constant (here 0.4$)$ and $\varepsilon \ll$ $u_{*} \kappa z_{0}$ is a small constant diffusivity that does not influence the solution in the ABL significantly. Here, we do not impose a specific value for $u_{*}$; it is found in an iterative approach by applying the log law at the first two nodes $\left(u_{*}=\kappa z d u / d z\right)$ where baroclinic departure from that law is insignificant (see profiles normalized by $u_{*}$ in the supplementary material). The chosen functional form of $\nu_{T}^{\text {tot }}$ complies with the LES setup, where a strong inversion layer is applied at $z>z_{i}$, resulting in negligible $\nu_{T}^{\text {tot }}$ magnitudes at such heights. Then, we substitute the proposed eddy viscosity formula [Eqs. (10)] into Eqs. (9) and solve the system of ODEs iteratively to obtain the velocity profiles. Note that in this reduced model, we do not tune any parameter when comparing with LESs, and the system of equations is closed.

Within the constant-flux layer (i.e., $z \leqq 0.1 z_{i}$ ), baroclinicity effects are weak, $\nu_{T}^{\text {tot }} \approx u_{*} \kappa z+O\left(\widetilde{z}^{2}\right)$ is a reasonable assumption (not shown), and model results show a good agreement with the corresponding LES profiles for both velocity components $(u, v)$. This can be seen from Fig. 4, which features a comparison between predictions from the proposed reduced model and LES profiles for all six considered cases (see Table 1).

Farther aloft (i.e., $z \gtrsim 0.1 z_{i}$ ), the dominant balance in Eqs. (9) is between the Coriolis forcing and the geostrophic wind vector [second and third terms on the lhs 


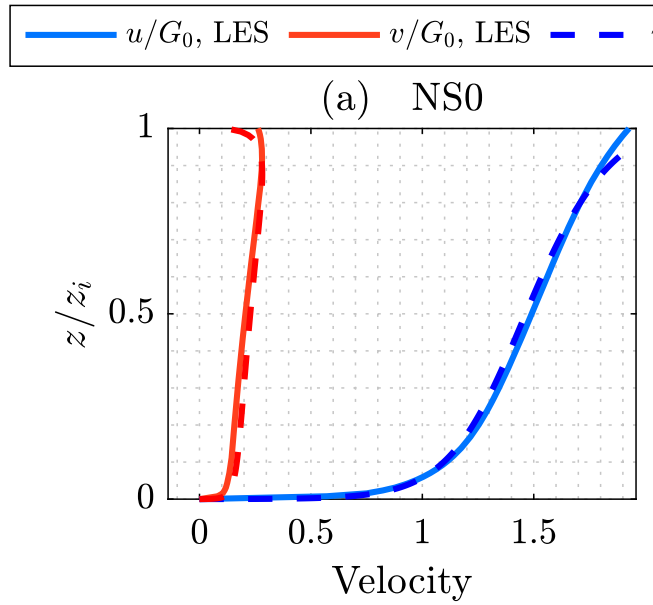
$u / G_{0}$, Reduced Model $-\quad v / G_{0}$, Reduced Model
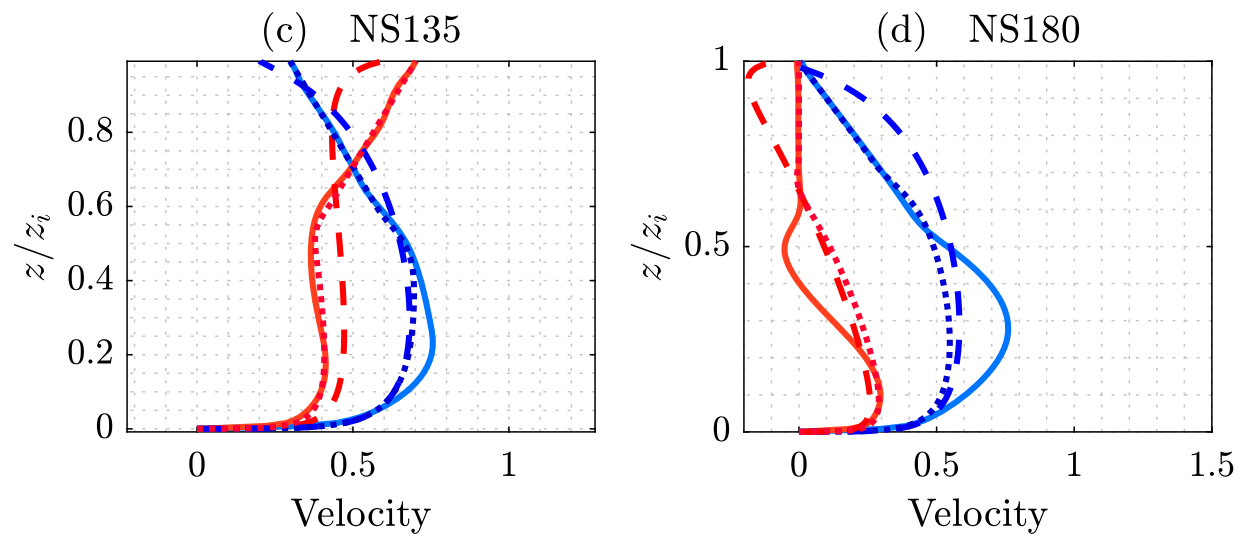

(e) NS270
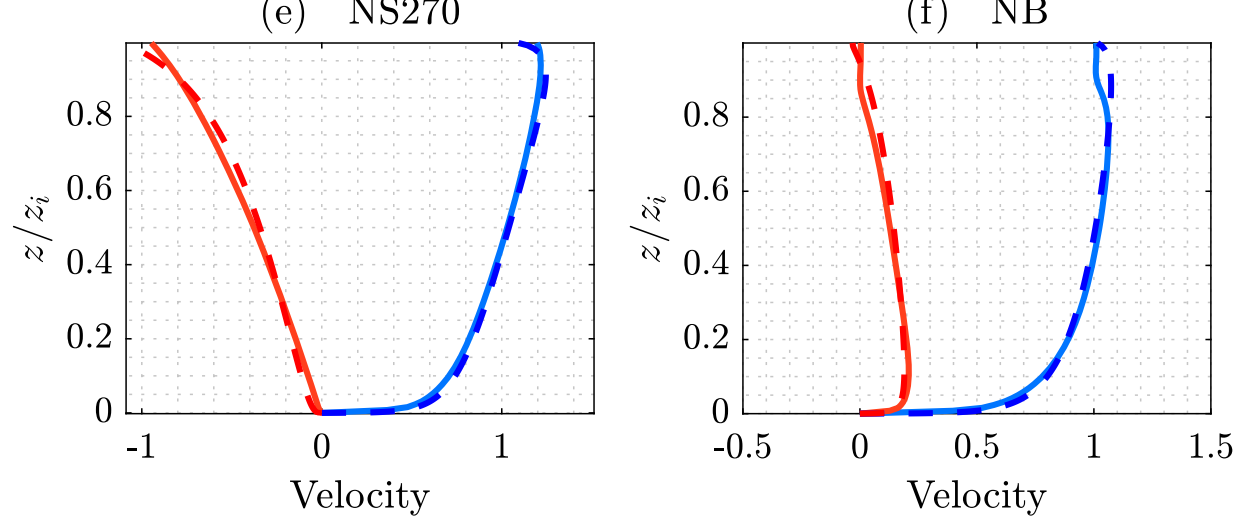

FIG. 4. Comparison of the simple reduced-order model (dashed lines) $u$ and $v$ profiles with the corresponding LES time-space-averaged profiles (solid lines). Blue lines denote $u$ and red lines denote $v$. (c),(d) The dotted lines represent the reduced-order model by decreasing the effective ABL depth in Eqs. (10) for eddy viscosity profiles by about $40 \%$.

of Eqs. (9)] for the positive geostrophic shear, cold and warm advection, and barotropic cases. As a result, at such heights, the momentum-flux divergence term [first term on the lhs of Eqs. (9)] is relegated to a minor momentummodulation role, and the governing equations are closed. It hence comes as no surprise that the simple reduced model is in a very good agreement with LES velocity profiles (Figs. 4a,b,e,f).
In the considered cases with substantial negative geostrophic shear, the reduction with height of the geostrophic wind vector modifies the dominant balance above the constant-flux layer, in favor of the stressdivergence term. In such conditions, a more accurate parameterization of turbulent fluxes is hence required along with a more refined definition of the effective top of the turbulent layer, which influences the viscosity 
profiles. Rather than using the height of the base of the capping inversion layer $z_{i}$, we use an effective ABL depth $z_{i \text {,eff }}=0.6 z_{i}$ in Eqs. (10) when applied to NS135 and NS180 cases (consistent with where TKE is significantly reduced, which will be discussed in the next section). The agreement of the reduced model (dotted lines) with the LES results is improved through the use of this effective depth. Nonetheless, the peak values of the jets were still not completely captured in the reduced model, which could be due to the chosen parameterization for turbulent fluxes. As described in the following section, the imposed negative geostrophic shear strongly impacts the momentum transfer coefficient above the constant-flux layer, reducing its magnitude via a reduction of the turbulence shear production. The O'Brien's eddy-viscosity model has a prescribed vertical structure and, not surprisingly, predicts a larger eddy viscosity at such heights, resulting in more diffused velocity profiles when compared to the reference LES ones.

Nevertheless, the important features of the baroclinic ABL are well captured across the considered cases (even without an accurate determination of the effective ABL depth), suggesting that this simple predictive model might be sufficient in many applications such as for estimating the baroclinicity effects on meteorological measurements and wind power applications. While we do not extend the model here to stable and unstable cases, this could be achieved via an alternative parameterization for $\nu_{T}$ that accounts for stability (and potentially a nonlocal flux term under unstable conditions). Further work will be required to account for the interacting effects of baroclinicity and stability on momentum transfer coefficients characterizing the ABL; these new eddy-viscosity parameterizations (e.g., considering a local eddy-viscosity formulation) will improve the predictive capabilities of such reduced-order models when stress divergence terms are relevant in the momentum-balance equations (e.g., in the NS180 case). To develop better turbulence closure models for baroclinic ABLs, one needs to understand how baroclinicity modulates the turbulence. Therefore, in the following section, we make a first step toward such future parameterizations, by quantifying the impact of baroclinicity and stability on turbulence statistics.

\section{c. Implications for turbulence}

Baroclinicity also strongly influences the turbulence characteristics of atmospheric flows, notably the TKE profiles. We report the total TKE (resolved plus SGS) profiles:

$$
\mathrm{TKE}=\bar{e}+e_{\mathrm{SGS}},
$$

where $\bar{e}=\overline{\tilde{u}_{i}^{\prime} \tilde{u}_{i}^{\prime}} / 2$ is the resolved and $e_{\mathrm{SGS}}=\sigma_{k k} / 2$ the SGS component of TKE (primes represent the turbulent fluctuations from the Reynolds average denoted by the overbar), the SGS contribution is estimated as

$$
e_{\mathrm{SGS}} \equiv \frac{L_{i i}}{2\left[(\hat{\Delta} / \tilde{\Delta})^{2 / 3}-1\right]},
$$

where $L_{i i}=\widehat{\widetilde{u_{i}}} \widehat{\widetilde{u}}_{i}-\widehat{\widetilde{u}}_{i} \widehat{\widetilde{u}}_{i}$ is the trace of the Leonard stress tensor; and $\hat{\Delta}$ and $\tilde{\Delta}$ are the widths of a test and a grid filter, respectively (Knaepen et al. 2002; Salesky and Chamecki 2012); we use $\hat{\Delta}=2 \tilde{\Delta}$.

In a steady barotropic neutral environment, the TKE decreases with height since its shear production also decreases [unsteady barotropic cases could be more complicated; e.g., Momen and Bou-Zeid (2016b)]. Our simulations illustrate that this is not necessarily the case in steady baroclinic ABLs. Figure 5 displays the TKE budget and TKE profiles in the neutral baroclinic cases. While the TKE decreases with height near the surface in all cases, it has a nonmonotonic trend farther aloft in some runs. For instance, it features an increase above the surface layer in the NS270 case (Fig. 5e).

The origin of the trends in the TKE profiles can be elucidated by examining the various terms in its budget equation. By assuming no subsidence, $\bar{w}=0$, and periodic statistical homogeneity in both horizontal directions, the conventional form of the resolved TKE budget can be obtained as follows (Momen and BouZeid 2017a):

$$
\begin{aligned}
\frac{\partial \bar{e}}{\partial t}= & -\overline{u^{\prime} w^{\prime}} \frac{\partial \bar{u}}{\partial z}-\overline{v^{\prime} w^{\prime}} \frac{\partial \bar{v}}{\partial z}+g \frac{\overline{w^{\prime} \theta^{\prime}}}{\bar{\theta}}-\frac{1}{\rho} \frac{\partial \overline{w^{\prime} p^{\prime}}}{\partial z} \\
& -\frac{\partial \overline{w^{\prime} e}}{\partial z}-\frac{\overline{\partial u_{i}^{\prime} \tau_{i 3}^{\prime}}}{\partial z}+\overline{\tau_{i j}^{\prime} S_{i j}^{\prime}},
\end{aligned}
$$

where $S_{i j}=1 / 2\left(\partial \tilde{u}_{i} / \partial x_{j}+\partial \tilde{u}_{j} / \partial x_{i}\right)$ is the strain-rate tensor. Note that all $u_{i}, p$, and $S_{i j}$ in Eq. (13) and the following equations are the resolved components but we henceforth omit the tildes for notational simplicity. We compute the TKE budget by defining the following expressions for the terms in Eq. (13) as follows:

$$
\mathrm{SP} \equiv-\overline{u^{\prime} w^{\prime}} \frac{\partial \bar{u}}{\partial z}-\overline{v^{\prime} w^{\prime}} \frac{\partial \bar{v}}{\partial z}=(\bar{u} \bar{w}-\overline{u w}) \frac{\partial \bar{u}}{\partial z}+(\bar{v} \bar{w}-\overline{v w}) \frac{\partial \bar{v}}{\partial z},
$$

$$
\mathrm{RT} \equiv-\frac{\partial \overline{w^{\prime} e}}{\partial z}=-\frac{1}{2} \frac{\partial}{\partial z}\left(\overline{u^{2} w}-2 \bar{u} \overline{u w}+\overline{v^{2} w}-2 \bar{v} \overline{v w}+\overline{w^{3}}\right)
$$

$\varepsilon_{\mathrm{SGS}} \equiv \overline{\tau_{i j}^{\prime} S_{i j}^{\prime}}=\overline{\tau_{i j} S_{i j}}-\overline{\tau_{i j}} \overline{S_{i j}}$, 


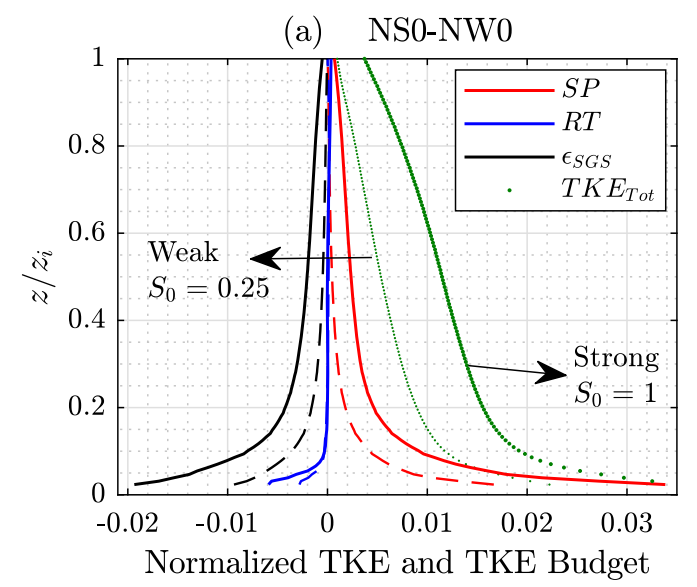

(c) NS135-NW135

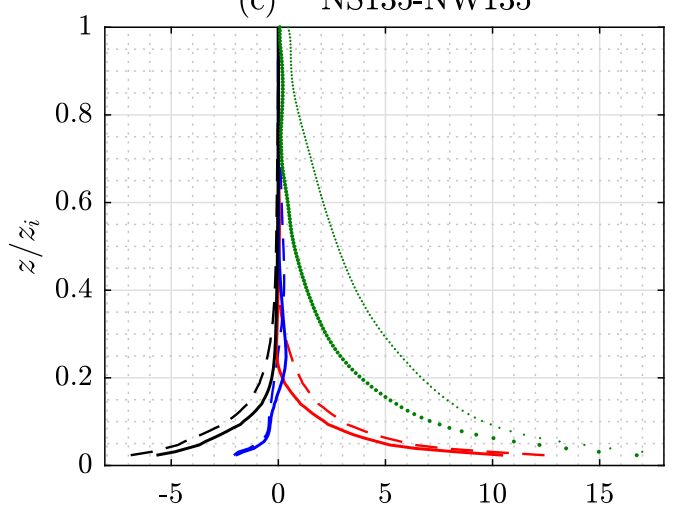

Normalized TKE and TKE Budget $\times 10^{-3}$

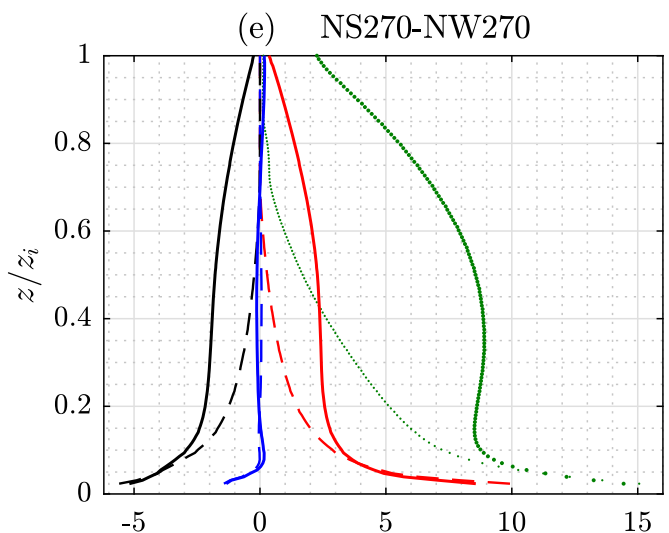

Normalized TKE and TKE Budget $\times 10^{-3}$

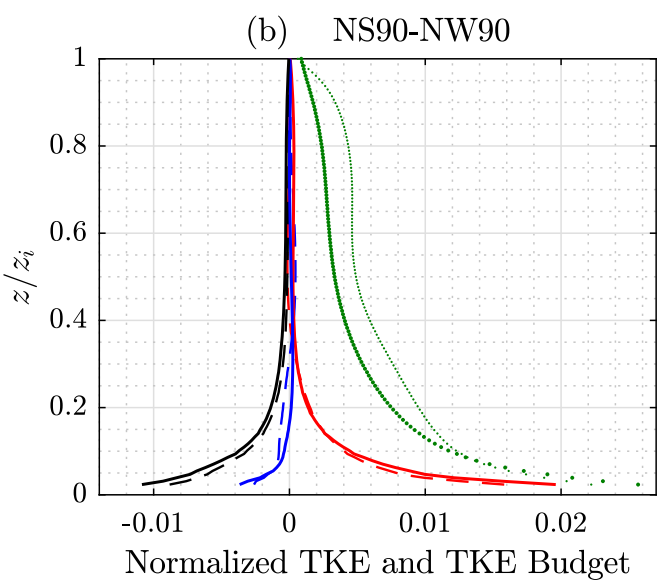

(d) NS180-NW180

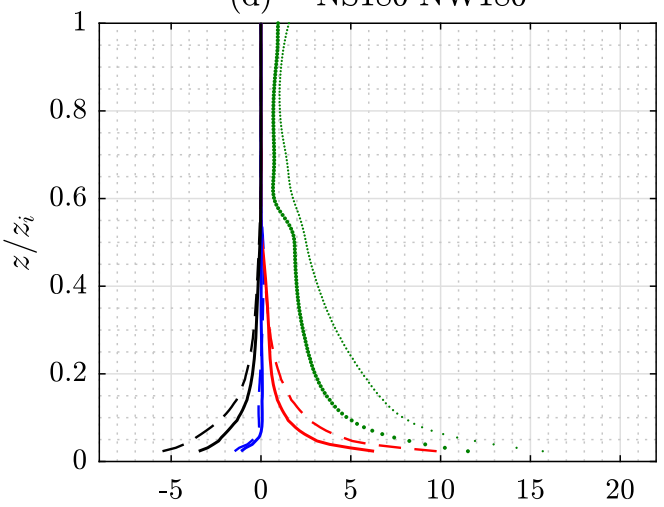

Normalized TKE and TKE Budget $\times 10^{-3}$

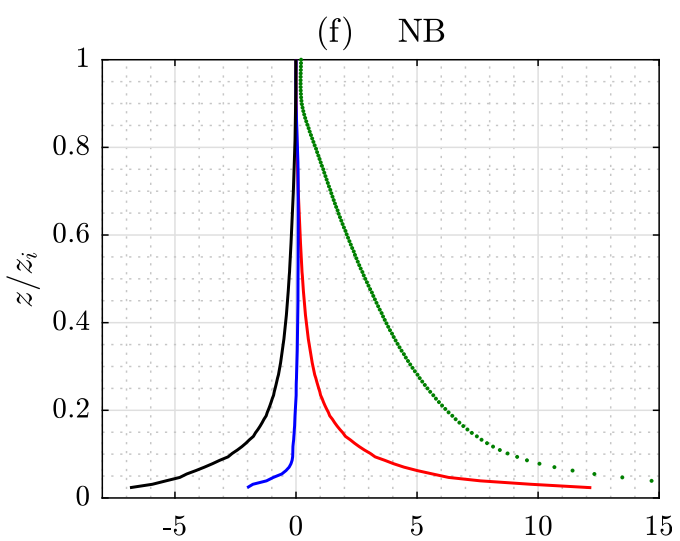

Normalized TKE and TKE Budget $\times 10^{-3}$

FIG. 5. The effect of baroclinicity strength and direction on the TKE budget and total TKE profiles. All the TKE budget terms are normalized with $z_{i} / G_{0}^{2}$ and the TKE profiles with $1 / G_{0}^{2}$. Solid lines show strong baroclinic cases and dashed lines indicate weak baroclinic cases. Budget terms are defined in Eqs. (13) and (14).

where SP, RT, and $\varepsilon_{\mathrm{SGS}}$ are, respectively, the shear production, resolved turbulent transport, and SGS dissipation [calculated, for better accuracy, using the expressions on the right-hand side of Eqs. (14) to avoid having to compute a perturbation during the LES run]. The other terms were found to be minor and are neglected here (the buoyancy term is not significant in the neutral ABLs away from top inversion layer, but will be included in the later analysis of the diabatic ABLs).

Positive geostrophic shear (Fig. 5a) significantly increases the magnitude of the budget terms. In the positive-shear case, $\mathrm{SP} \gg \mathrm{RT}$ inside the ABL $\left(0<z / z_{i}<1\right)$ since the imposed 
pressure gradient produces a constant shear in height. However, in the barotropic case (Fig. 5 f), $1 \lesssim \mathrm{RT} / \mathrm{SP} \lesssim 1.5$ for $0.7<z / z_{i}<1$. On the other hand, in the cases with a negative geostrophic shear (Figs. 5c,d) the magnitude of the shear production decreases relative to the barotropic reference.

In summary, the magnitude of the TKE budget terms in all the neutral cases is altered by the baroclinicity direction and strength. Their shapes are equally influenced. For instance, we see increasing values of TKE profiles with height at some levels in NS270 (Fig. 5e). We also observe an inflection point in the SP and $\varepsilon_{\text {SGS }}$ profiles of NS270, which is not seen in the barotropic case.

\section{The interacting effects of baroclinicity and stability}

In this section, we describe how baroclinicity impacts the ABL mean and turbulence behavior in the presence of buoyancy forces. To that end, we only show the strong baroclinic ABLs $\left(S_{0}=1\right)$ under the imposed surface heat fluxes $\left(+200\right.$ and $\left.-20 \mathrm{~W} \mathrm{~m}^{-2}\right)$ to distinguish their effects more clearly from the barotropic cases.

\section{a. Implications for the mean velocity}

Baroclinicity strongly affects the shape of the wind speed profiles in the diabatic ABLs, as illustrated in Fig. 6. Unconventional velocity profiles are clearly observed in this figure. For example, negative geostrophic shear can lead to velocity profiles that decrease with height (Figs. 6c and 6d) (e.g., over more than half of the effective ABL depth in neutral cases). The positiveshear case also highly increases the magnitude of the wind speeds (more than 50\% compared to the barotropic environment with the same ground-level geostrophic wind).

Away from the surface layer, $z / z_{i}>0.3$, the turbulent friction force is decreased. Thus, in a neutral ABL, the dominant balance would be determined by Coriolis and pressure gradient forces, resulting in geostrophic winds in that region. Hence, it is expected that the wind velocities will align with the geostrophic wind vector in the outer layer (see Fig. 6). Buoyancy forces alter this balance. As Fig. 6 shows, stable cases have the closest match between the geostrophic and actual wind speed profiles, while winds in the unstable cases result in strong departures from their geostrophic counterparts. This is attributed to the negative stabilizing heat fluxes in the stable cases that damp the turbulence in the $\mathrm{ABL}$ and reduce friction. Hence, the main balance between Coriolis force and geostrophic wind in the outer layer is more dominant, with minimal frictional effects. Nevertheless, in the unstable cases, friction is increased, and the vertical mixing is enhanced. Therefore, the profiles are more uniform, and they depart from the geostrophic winds more than other cases.

Baroclinicity also impacts the much-studied low-level jet (LLJ) characteristics in the stable ABL. The LLJ is defined as a region inside ABL where the wind speed is significantly faster than wind speeds above it (Stull 1988). In the stable barotropic ABL, a weak LLJ is formed at the height where the TKE is significantly weakened (Fig. 6f). To assess the LLJ behavior, we define the LLJ location $Z_{\mathrm{LLJ}}$ and its strength $S_{\mathrm{LLJ}}$ as follows:

$$
\begin{aligned}
\frac{\partial \bar{M}}{\partial z}\left(Z_{\mathrm{LLJ}}\right) & =0 \quad \text { and } \quad \frac{\partial^{2} \bar{M}}{\partial z^{2}}\left(Z_{\mathrm{LLJ}}\right)<0 ; \\
S_{\mathrm{LLJ}} & \equiv \frac{\bar{M}\left(Z_{\mathrm{LLJ}}\right)}{\left|G\left(Z_{\mathrm{LLJ}}\right)\right|} .
\end{aligned}
$$

The strength of LLJ for a barotropic case is $S_{\mathrm{LLJ}, \mathrm{SB}} \approx$ 1.1. This strength is decreased when there is a positive shear $\left(S_{\text {LLJ,SS0 }}=1.05\right.$, Fig. $\left.6 \mathrm{a}\right)$ and intensified when there is a negative shear $\left(S_{\mathrm{LLJ}, \mathrm{SS} 135}=1.11\right.$, Fig. $6 \mathrm{c}$, and $S_{\text {LLJ,SS180 }}=1.14$, Fig. $6 \mathrm{~d}$ ). We also observe jetlike profiles in neutral and unstable conditions (albeit weaker than under stable conditions) when there is a negative shear (Figs. $6 \mathrm{c}$ and $6 \mathrm{~d}$ ). The LLJ location, $Z_{\mathrm{LLJ}}$, is also influenced by the baroclinicity direction $\beta$. A positive geostrophic shear and a warm advection tend to elevate it while a negative shear lowers its height. The LLJ location and strength are very important for the windenergy industry. LLJs have the potential to boost the wind power production in wind farms and lead to higher economic benefits for wind-farm owners [see, e.g., Nunalee and Basu (2014) for LLJ implications in windenergy industry]. Hence, our results could be useful for wind-energy applications (such as site selection) to better understand how the LLJ location and strength are modulated by baroclinicity in the atmosphere. Note that these results are not only valid for the wind speed profiles nondimensionalized by the geostrophic wind vector at the surface, $G_{0}$, but also, they show similar characteristics if normalized by the height-varying geostrophic wind vector, $G(z)$ (as shown in the supplemental material, although the LLJ is not typically defined in this way in the literature).

The Ekman spirals are similarly altered significantly by the presence of the baroclinicity. Figure 7 displays the hodographs for baroclinic and barotropic ABLs under stable and unstable conditions. The wind profiles in unstable cases do not converge to the geostrophic values within the ABL, which lie on the dotted line, because of the significant friction (turbulent stresses) at all heights. They converge to geostrophic values after the capping inversion layer starts and damps the shear stress. As expected, the hodographs in unstable conditions are 


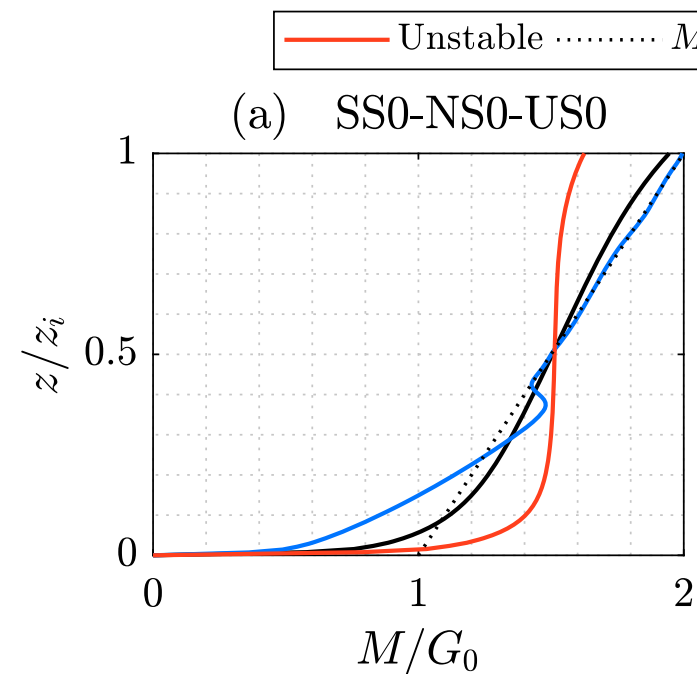

$M_{g} / G_{0}$

Stable

Neutral
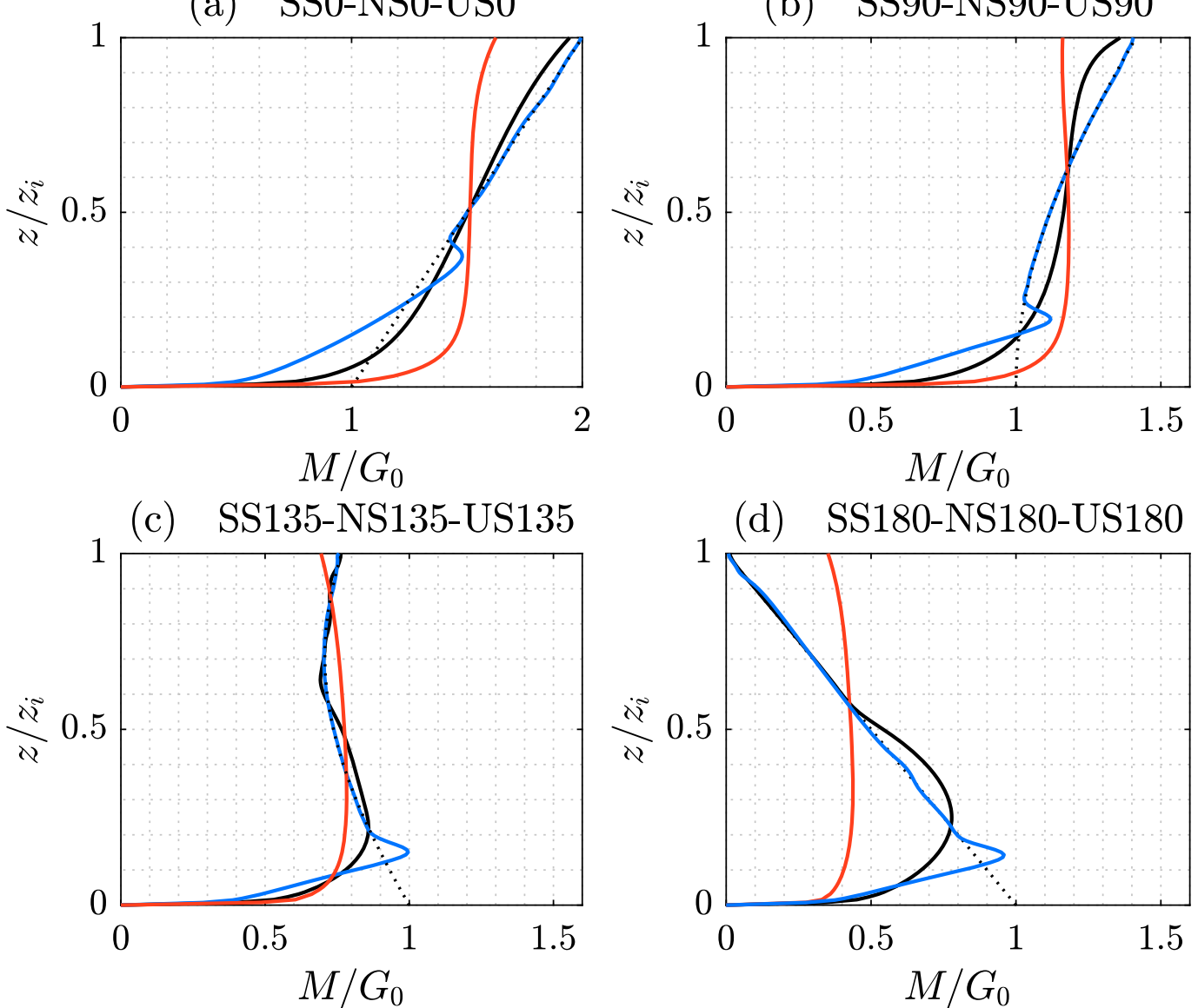

(e) SS270-NS270-US270
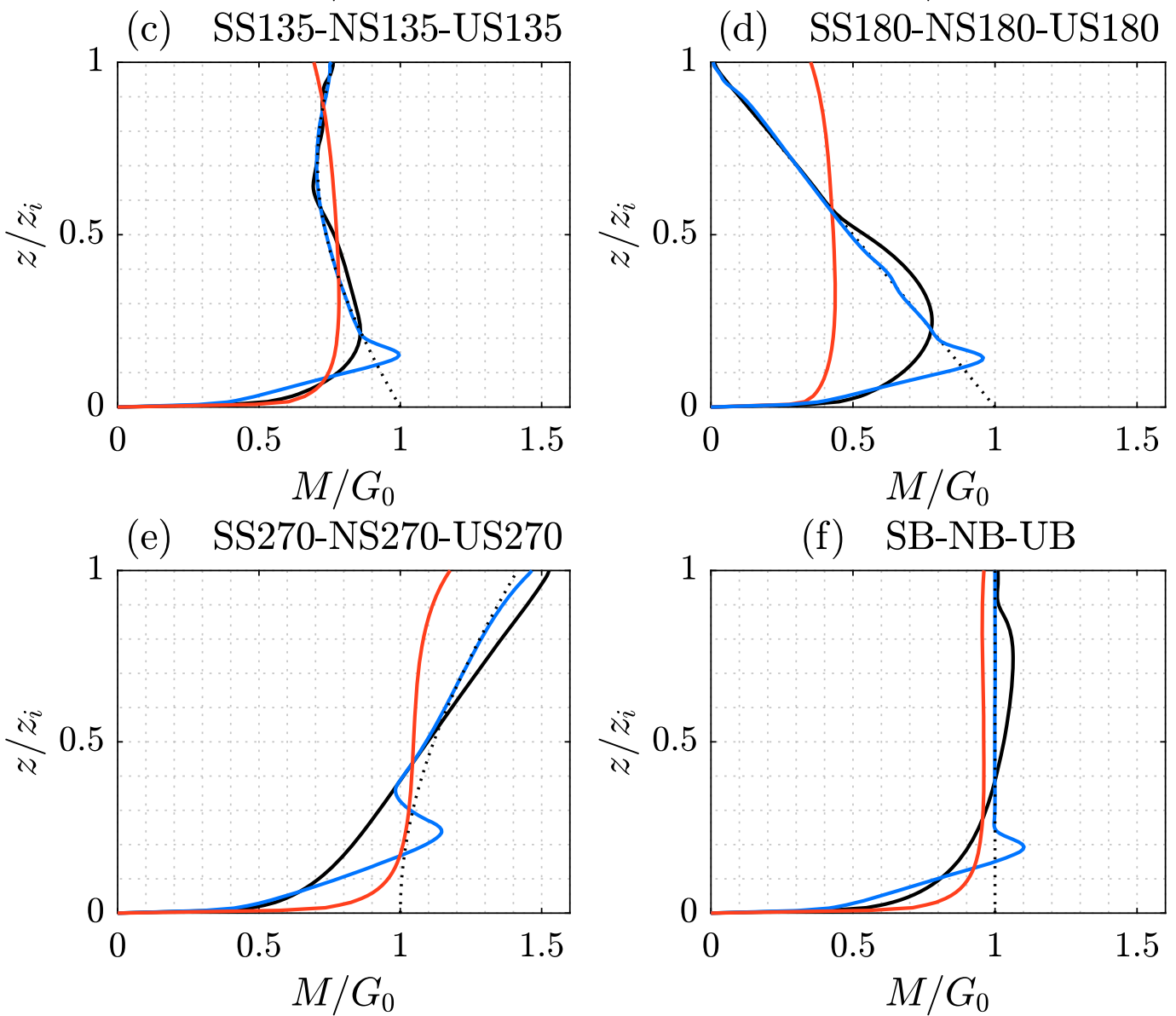

FIG. 6. The interacting effect of stability and baroclinicity: dimensionless wind speed profiles of baroclinic and barotropic ABLs under stable, neutral, and unstable conditions. The dotted lines represent the corresponding geostrophic wind magnitude in height.

contracted since the mixing and vertical coupling are high, leading to more uniform velocity profiles. On the other hand, in stable conditions eccentric hodographs are observed due to weaker mixing and vertical coupling. The velocity vectors change more significantly in baroclinic cases with height compared to their barotropic counterpart. As noted in the introduction, these modifications will alter the Ekman pumping process and influence the genesis of 


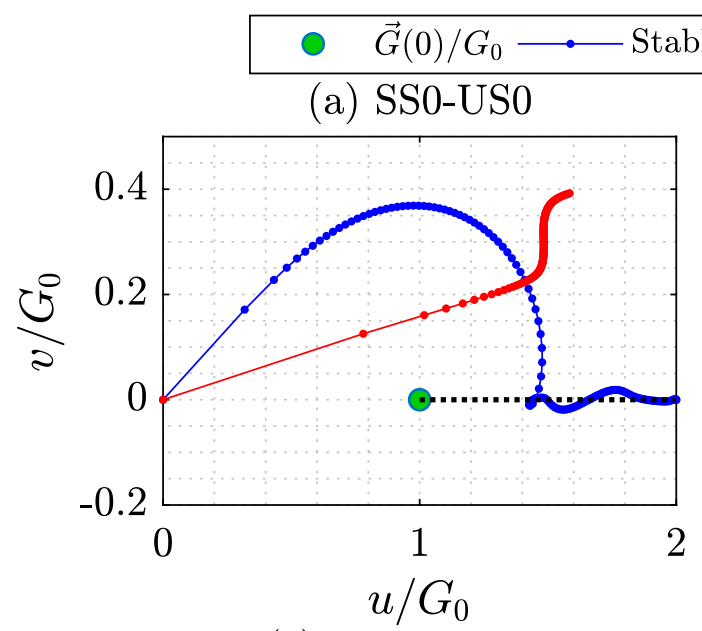

(c) SS135-US135

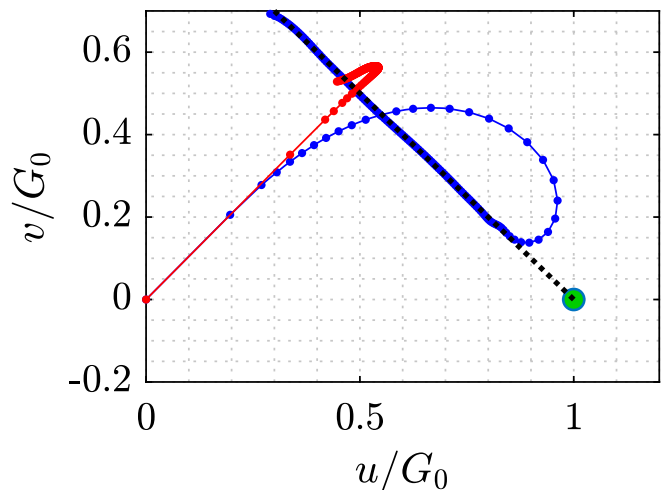

(e) SS270-US270

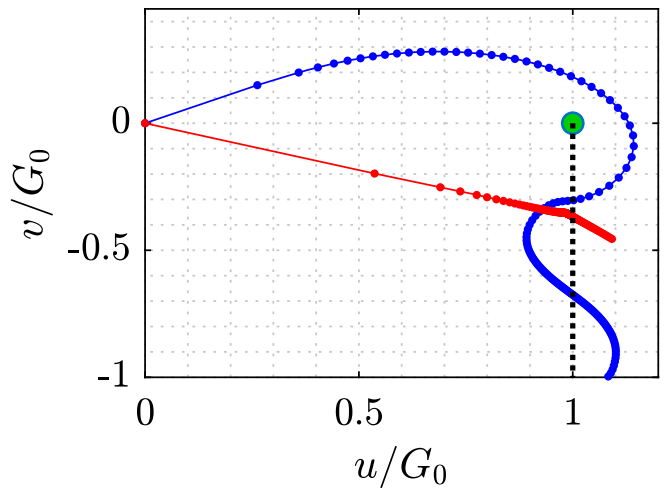

$\vec{G}(z) / G_{0}$

(b) SS90-US90

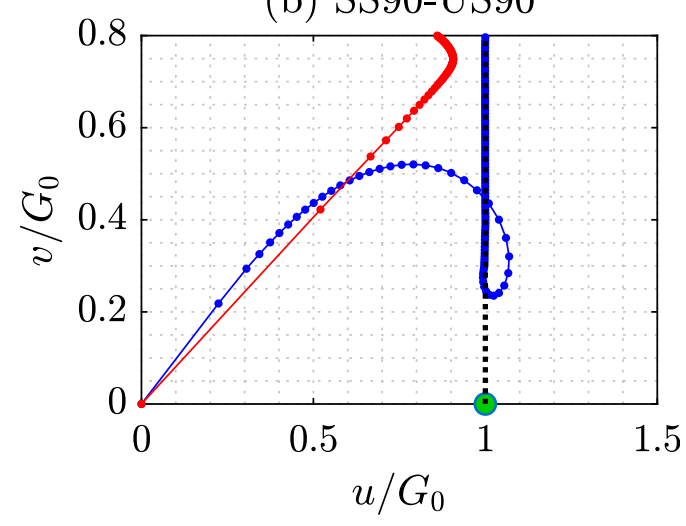

(d) SS180-US180

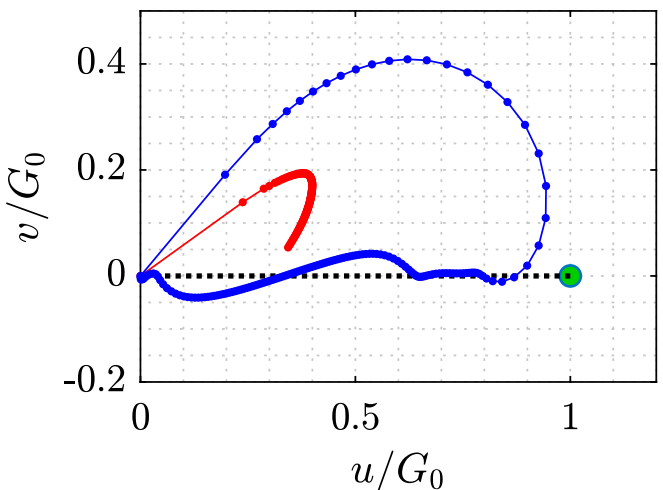

(f) SB-UB

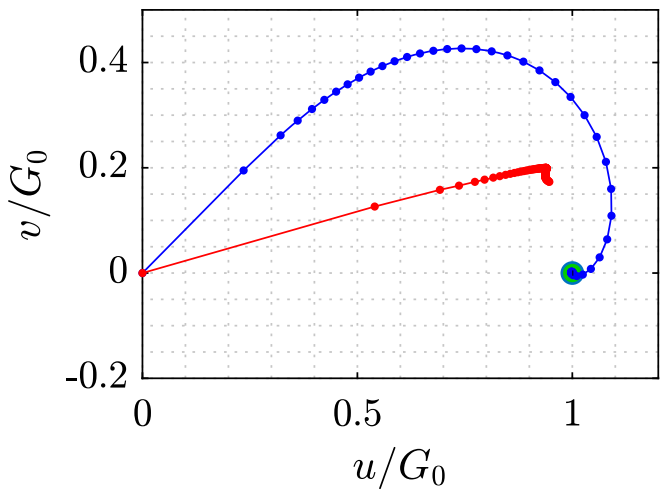

FIG. 7. Velocity hodographs for all stable and unstable cases. The unstable ABLs have more uniform profiles and hence their hodographs are shorter, whereas the hodographs of the stable simulations are more spread because of stronger vertical decoupling and lower mixing. The black dotted lines indicate the geostrophic-wind vectors (for strong baroclinicity). The points with $(u, v)=(0,0)$ denote the surface $z=z_{0}$ for the velocity plots, while the green dots represent the surface geostrophic velocity vector.

storms and fronts. Understanding the genesis of storms and their associated precipitation by baroclinicity are crucial for models that aim to parameterize hydrometeorological processes and land-atmosphere interactions (e.g., Ramos da Silva and Avissar 2006; Momen et al. 2017a). This occurs because of variations in the cross-isobaric mass transport and mass flux out of the boundary layer in low and high pressure systems. For instance, the cross-isobaric mass transport, $\mathrm{CMT} \equiv 1 /\left(z_{i} G_{0}\right) \int_{z=0}^{z=z_{i}} v(z) d z$, in our barotropic cases $\left(\mathrm{CMT}_{\mathrm{SB}}=0.07, \mathrm{CMT}_{\mathrm{UB}}=0.19\right.$; see Fig. $\left.7 \mathrm{f}\right)$, is significantly increased in the positive geostrophic shear $\left(\mathrm{CMT}_{\mathrm{SS} 0}=0.61, \mathrm{CMT}_{\mathrm{US} 0}=0.77\right.$; see Fig. 7a) and slightly 
TABLE 2. The impact of baroclinicity, static stability, and their interactions on the friction velocity defined as follows: $u_{*}=$ $\left\{\left[(\overline{\tilde{u} \tilde{w}}-\overline{\tilde{u}} \overline{\tilde{w}})+\tau_{x z}\right]^{2}+\left[(\overline{\tilde{v} \tilde{w}}-\overline{\tilde{v}} \overline{\tilde{w}})+\tau_{y z}\right]^{2}\right\}^{1 / 4}$. Here $L=-u_{*}^{3} \bar{\theta} / \kappa g w^{\prime} \theta^{\prime}$ is the Obukhov length based on the values of the parameters at the surface, $z_{i}$ is the imposed inversion height (1000 $\mathrm{m}$ for unstable cases and $800 \mathrm{~m}$ for stable cases), and $\kappa$ is the von Kármán constant (here 0.4 ).

\begin{tabular}{|c|c|c|c|c|c|c|}
\hline \multirow{2}{*}{$\begin{array}{l}\text { Simulation name (baroclinicity strength } \\
\text { and direction; see Table 1) }\end{array}$} & \multicolumn{3}{|c|}{$u_{*}\left(\mathrm{~m} \mathrm{~s}^{-1}\right)$} & \multicolumn{3}{|c|}{$z_{i} / L$} \\
\hline & Stable (S) & Neutral (N) & Unstable (U) & Stable (S) & Neutral (N) & Unstable (U) \\
\hline$* \mathrm{~B}$ & 0.28 & 0.37 & 0.53 & 7.6 & 0.0 & -14.1 \\
\hline$* \mathrm{~W} 0$ & & 0.41 & & & 0.0 & \\
\hline *W90 & & 0.40 & & & 0.0 & \\
\hline *W135 & & 0.37 & & & 0.0 & \\
\hline *W180 & & 0.35 & & & 0.0 & \\
\hline *W270 & & 0.35 & & & 0.0 & \\
\hline *S0 & 0.34 & 0.51 & 0.73 & 4.3 & 0.0 & -5.4 \\
\hline$* \mathrm{~S} 90$ & 0.29 & 0.44 & 0.62 & 6.9 & 0.0 & -8.8 \\
\hline$* \mathrm{~S} 135$ & 0.26 & 0.36 & 0.47 & 9.5 & 0.0 & -20.2 \\
\hline$* \mathrm{~S} 180$ & 0.25 & 0.30 & 0.31 & 10.7 & 0.0 & -70.2 \\
\hline$* \mathrm{~S} 270$ & 0.27 & 0.32 & 0.52 & 8.5 & 0.0 & -14.5 \\
\hline
\end{tabular}

decreased in the negative geostrophic shear $\left(\mathrm{CMT}_{\mathrm{SS} 180}=\right.$ $0.05, \mathrm{CMT}_{\mathrm{US} 180}=0.15$; see Fig. $7 \mathrm{~d}$ ).

\section{b. Implications for turbulence}

The friction velocity is highly modulated by the baroclinicity strength and through the interaction of baroclinicity and stability. Table 2 indicates that increasing surface heat flux from stable to neutral and to unstable increases the friction velocity as expected since the turbulence intensity rises. Furthermore, as the strength of the baroclinicity in neutral cases increases the changes in the friction velocity compared to the barotropic case become higher. In general, irrespective of the static stability in the diabatic cases and the baroclinicity strength in neutral simulations, the friction velocity in the positive geostrophic shear and coldadvection cases increases compared to the barotropic environment, while it decreases in the cases with negative geostrophic shear. These variations in the friction velocity are more pronounced in the unstable cases than in the neutral and stable cases since under unstable conditions mixing and vertical coupling are high and the surface is thus more influenced by the imposed geostrophic wind shear at higher elevations. On the other hand, in stable cases the surface is more decoupled from the middle and top of the ABL and hence it is not significantly affected by the changes in the geostrophic wind vector away from the surface.

The ratio of the shear production over the buoyant production or destruction, which is the negative inverse of the flux Richardson number $\left(-\mathrm{Ri}_{f}^{-1}\right)$, is calculated to infer how it is modulated by baroclinicity. $\mathrm{Ri}_{f}$ is a dimensionless number that indicates the dominance of buoyant versus shear production of turbulence in the $\mathrm{ABL}$ and $\mathrm{Ri}_{f} \equiv-\mathrm{BP} / \mathrm{SP}$, where $\left.\mathrm{BP} \equiv g \bar{\theta}^{-1} \overline{\left(w^{\prime} \theta^{\prime}\right.}+\pi_{3}\right)=$ $g \bar{\theta}^{-1}\left[(\overline{w \theta}-\bar{w} \bar{\theta})+\pi_{3}\right]$ is the total buoyant production or loss. Here, to get the BP, we add the SGS contribution to the resolved component. Additionally, this number can characterize the local stability of the flow. In the unstable barotropic simulation (Fig. 8f), SP is almost 2 times BP very close to the surface, while above $z / z_{i}>$ $0.1, \mathrm{BP}$ is dominant. In the stable barotropic case (Fig. 8f), SP reaches a minimum in proximity of the LLJ where it becomes slightly negative-often corresponding to a global energy backscatter region-that indicates energy transfer from small to large scales [conversion of TKE to mean kinetic energy; e.g., Bou-Zeid et al. (2009); Gayen et al. (2010)]. This negative shear production occurs right above ;the LLJ, where $d U / d z<0$ and $\overline{u^{\prime} w^{\prime}}<0$ (see, e.g., the TKE budget of stable cases in the supplemental material).

Near the surface and for a constant surface buoyancy flux, baroclinicity alters the stability of the flow. Positive geostrophic shear and cold advection tend to increase the $\mathrm{Ri}_{f}^{-1}$ magnitude, which implies that the ABL is closer to neutral conditions. Conversely, a negative geostrophic shear tends to decrease the $\mathrm{Ri}_{f}^{-1}$ magnitude, which implies that the ABL becomes more unstable. This fact is also clear from the $z_{i} / L$ values shown in Table 2 (cf. these cases with their barotropic counterpart). However, away from the surface, $0.3<$ $z / z_{i}<1$, baroclinicity always increases shear production in the $\mathrm{ABL}$ and tends to make the outer $\mathrm{ABL}$ flow more neutral throughout the considered cases by increasing $\left|\mathrm{Ri}_{f}^{-1}\right|$. This can be related to the geostrophic shear that results in wind shear and turbulence production even far from the surface in all baroclinic cases, but not in the barotropic one. Note that this outer region in the stable ABL corresponds to the flow above the LLJ, which is completely decoupled from the surface and often not considered part of the stable ABL. 
(a) SS0-US0

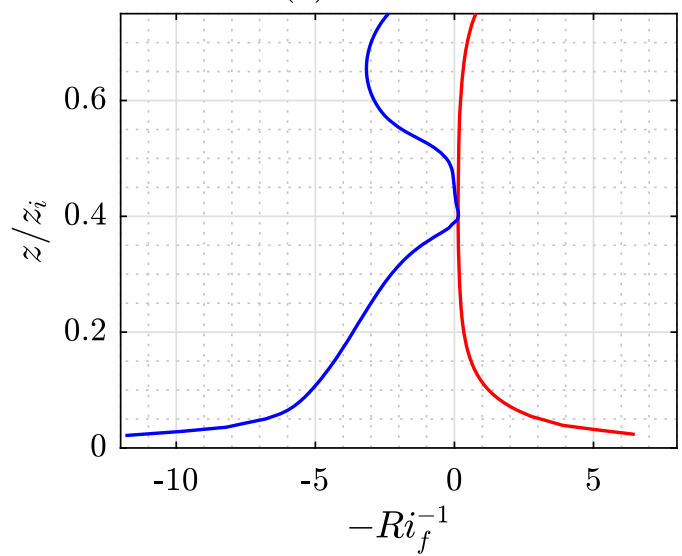

(c) SS135-US135

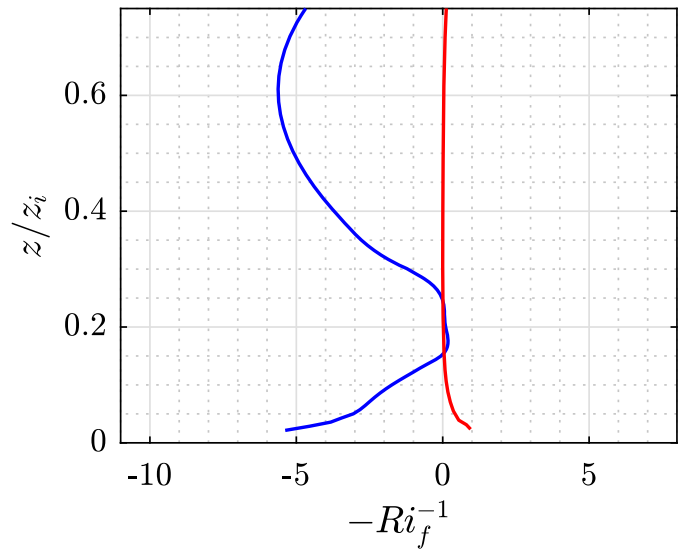

(e) SS270-US270

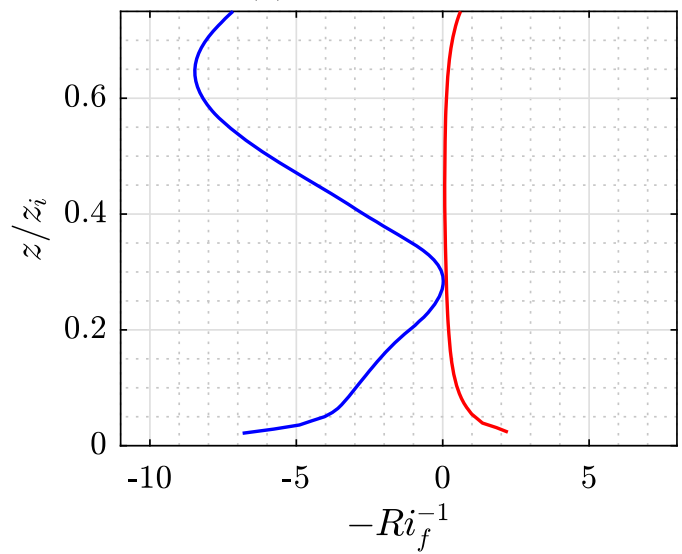

(b) SS90-US90

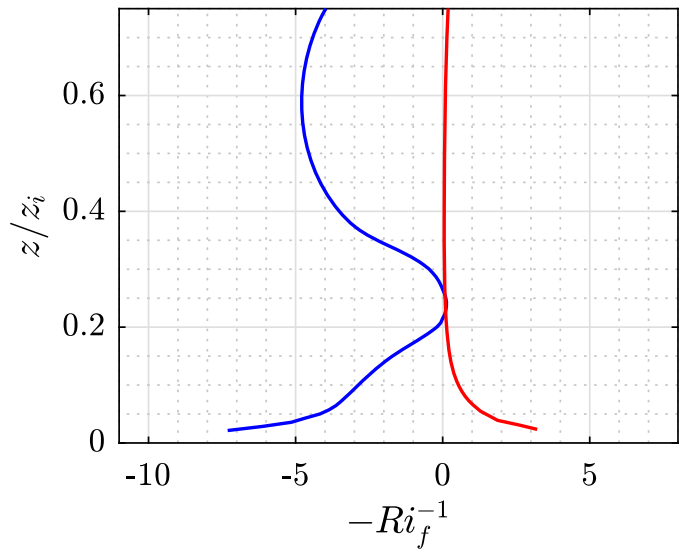

(d) SS180-US180

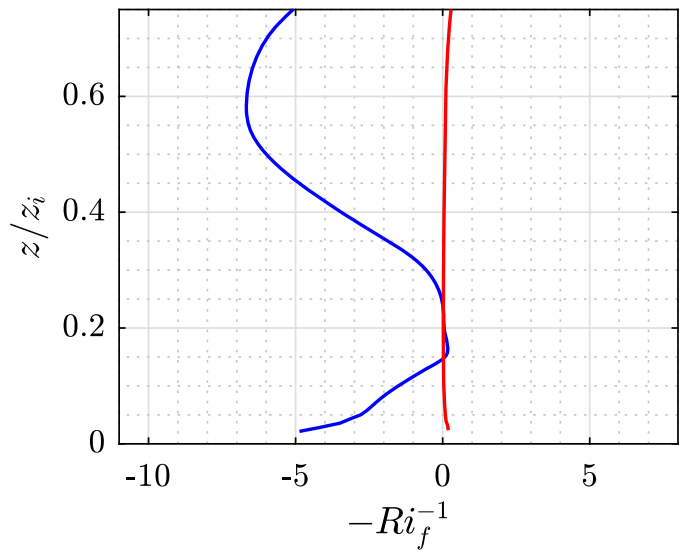

(f) $\quad$ SB-UB

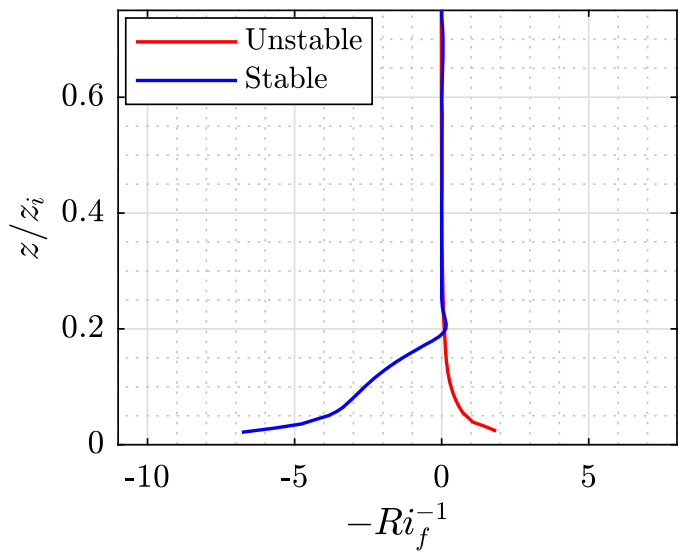

FIG. 8. The ratio of the shear production over buoyant production in the presence of strong baroclinicity. The magnitudes are largely influenced by the baroclinicity in both stable and unstable conditions and the profile shapes are remarkably distinct from (f) their barotropic counterparts.

In the stable baroclinic cases, the departures of $\mathrm{Ri}_{f}^{-1}$ from the barotropic reference very close to the surface are weaker than at higher elevations. That is because in stable environments the vertical profiles are more decoupled from the wall. Therefore, the surface value is mainly controlled by the imposed pressure gradient at or near the wall, which is equal to the barotropic geostrophic value, rather than the pressure gradient aloft. However, at higher heights, $0.3<z / z_{i}<1, \mathrm{Ri}_{f}^{-1}$ changes substantially compared to the barotropic 
case. In the stable barotropic ABL, the ratio of the shear over buoyancy is near zero at higher elevations, $0.3<z / z_{i}<1$. However, in the baroclinic environments, this ratio becomes $<-1$ and the ABL is hence shear dominated above the LLJ.

The baroclinic unstable environments show significant $\mathrm{Ri}_{f}^{-1}$ departures from their barotropic counterpart near the surface since mixing is high and thus the surface is more influenced by changes at higher elevations. In the unstable positive-shear case (Fig. 8a), the shear over buoyant production ratio $\mathrm{Ri}_{f}^{-1}$ becomes about 3 times larger than the barotropic case (Fig. 8f) near the surface. At higher elevations, $0.1<z / z_{i}<0.8$, unlike the barotropic unstable case and all stable cases, shear increases and the buoyancy term remains dominant.

The TKE profiles and budgets, illustrated in Fig. 9, show that in the positive-shear case US0, the TKE becomes relatively constant in the middle of the domain because of the persistent imposed geostrophic shear. All the TKE budget terms are magnified in this case (Fig. 9a) compared to the barotropic case (Fig. 9f). Moreover, $\mathrm{RT} / \varepsilon_{\mathrm{SGS}}$ becomes smaller than under the barotropic condition indicating that the relative importance of turbulent transport over dissipation decreases and production-dissipation balance is more applicable (so the produced turbulence at the surface in case US0 is transported upward less than in case UB). As in the neutral cases, the TKE displays some unusual vertical trends in the baroclinic diabatic ABLs such as increasing magnitude with height, Fig. 9d for $0.15<z / z_{i}<0.35$, under unstable condition (as well as in stable cases SS180 and SS270 shown in the supplemental material).

In summary, baroclinicity significantly impacts the magnitude of the TKE and TKE budget terms, changes their relative ratios in the ABL, and alters their profiles. These results indicate that it is imperative to consider baroclinicity effects in future ABL models and turbulence closures in order obtain a more accurate representation of real-world ABLs. This can be done by employing $S_{0}$ and $\beta$ parameters [Eq. (8)] in such models, in addition to conventional parameters such as friction velocity, aerodynamic roughness length, surface heat flux, and ABL height. Local eddy viscosity models might be able to account indirectly for the effect of $S_{0}$ and $\beta$ if they can dynamically capture the resulting changes in mean wind magnitude and direction related to baroclinicity (i.e., as in the analytical model we presented for the neutral cases), but for nonlocal models this might be more challenging.

\section{c. Implications on the large turbulence structures}

The coherent turbulence structures are significantly influenced by the baroclinicity since it increases or reduces shear production in the ABL. In particular, here we present the effects on convective ABLs. In such flows, cellular- or roll-type convections in the velocity fields are formed. Certain environments can produce wide or narrow mixed-layer rolls and they modulate large-scale motions and thermals, potentially in a twoway coupling (Young et al. 2002). These thermal convection structures, under different stabilities, can lead to instability in the Ekman boundary layer (Lilly 1966). Most of the previous studies focused on the role of the stability parameters like $z_{i} / L$ (Deardorff 1972; Salesky et al. 2017) in favoring rolls or cells regimes. We show here how these coherent ABL structures are also modulated by baroclinicity, at a constant surface heat flux.

Figure 10 displays the normalized instantaneous vertical velocity field in three convective cases at two different heights. Figures 10a and 10b show the barotropic simulations whereas Figs. 10c and 10d display the warmadvection case and Figs. 10e and 10f depict the negative geostrophic shear case. Figures 10a-d exhibit the signature of the well-documented streamwise-elongated, high and low momentum bulges (red strips of high velocity, often referred to as the large-scale motions) whereas Figs. 10e and 10f indicate convective cells in the negative geostrophic shear case (see also the supplemental material). Salesky et al. (2017) found that the transition from rolls to cells occurs in the regions where $z_{i} / L \approx-15$ to -20 . From Table 2 , one can note that the UB and US270 cases depicted in Fig. 10 have very similar values of $z_{i} / L \approx-14$. This explains the similarity of their roll structures. Case US180 on the other hand has $z_{i} / L=-70.2$, placing it in the cell region according to Salesky et al. (2017); this is indeed confirmed in Fig. 10. Hence, baroclinicity can lead to the formation of convective cells even when the heat flux and geostrophic wind values at the surface suggest the presence of rolls. This modulation appears to be consistent with the modification of $z_{i} / L$ and the transition from rolls to cells seems to occur at $z_{i} / L$ values comparable to the barotropic ABL. Nevertheless, even at the same $z_{i} / L$ of a barotropic counterpart, baroclinicity will change the direction of the mean flow with height, with apparent consequences on the large-scale motions. For instance, Figs. $10 \mathrm{c}$ and $10 \mathrm{~d}$ indicate that the rolls are inclined laterally (cf. it with the barotropic case shown in Figs. 10a and 10b) because of the imposed geostrophic wind vector in the negative $y$ direction.

\section{Conclusions}

We investigated the baroclinic environment, which emerges when the isobars intersect the isotherms and isopycnals, and which occurs very frequently in the 
(a) US0

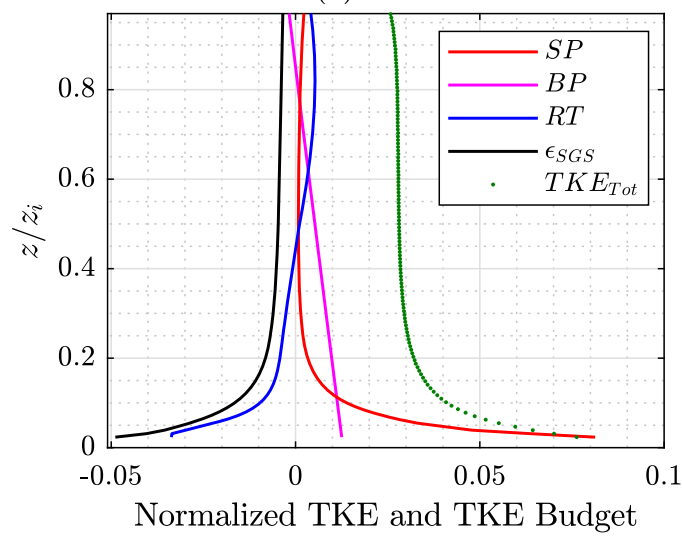

(c) US135

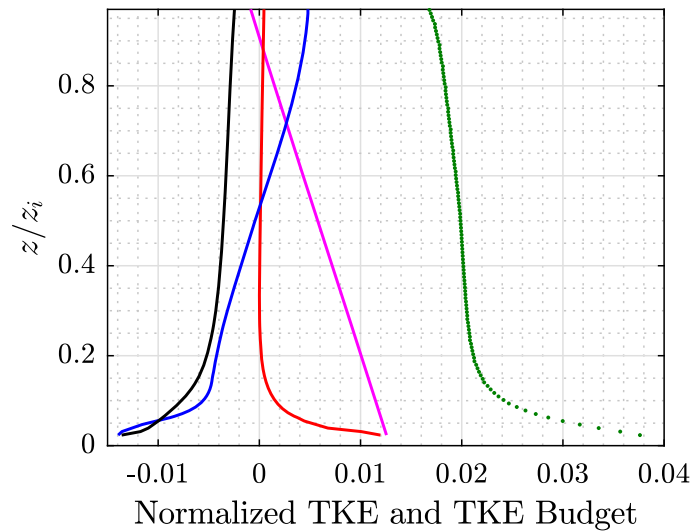

(e) US270

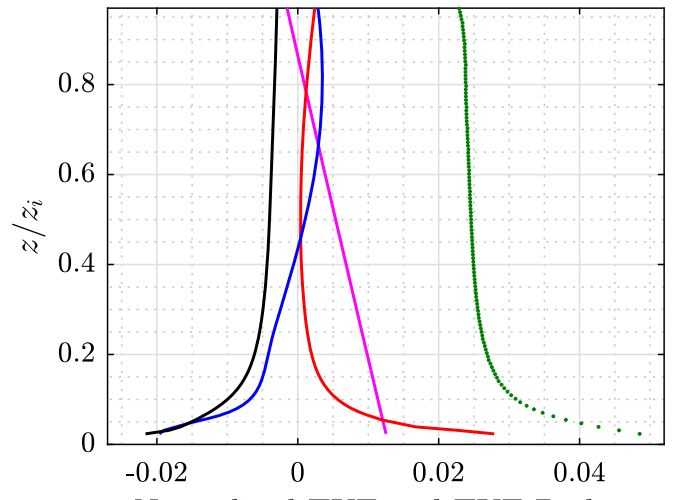

Normalized TKE and TKE Budget (b) US90

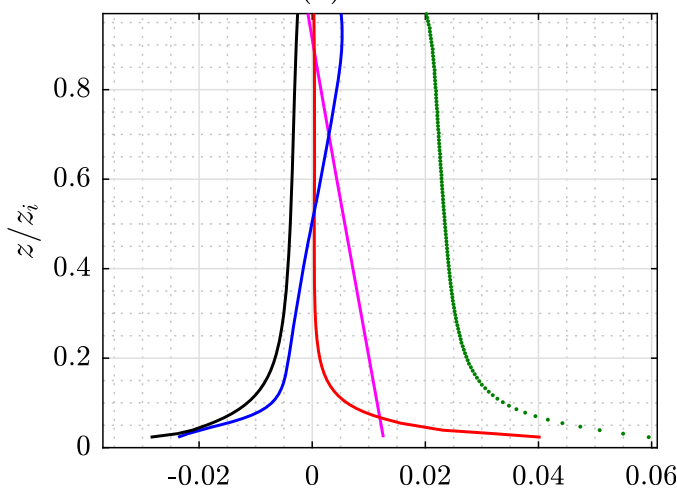

Normalized TKE and TKE Budget

(d) US180

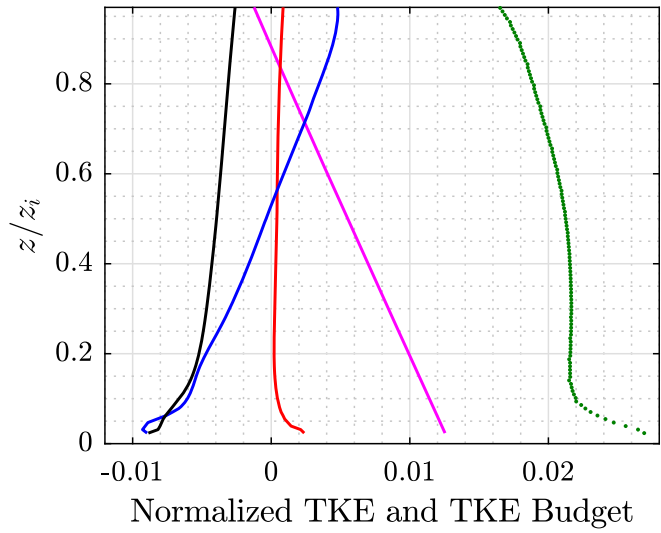

(f) UB

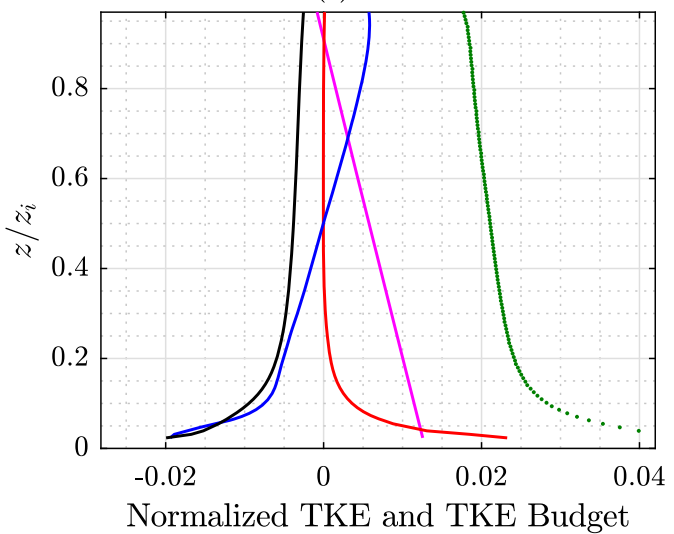

FIG. 9. The interacting effects of baroclinicity and stability on the TKE budget and TKE profiles. All the TKE budget terms are nondimensionalized by $z_{i} / G_{0}^{2}$ and the TKE profiles are rescaled with $1 / G_{0}^{2}$. This figure shows all the unstable cases (the profiles of the stables cases can be found in the supplemental material).

real-world atmospheric boundary layer because of the prevalent strong horizontal temperature gradients. Unlike the barotropic case, both the magnitude and direction of the pressure gradient vector can change in height in a baroclinic environment. We introduced two parameters to quantify the baroclinicity strength $S_{0}$ and direction $\beta$, similar to Arya and Wyngaard (1975), based on the thermal wind vector components. Each of them can have various distinctive impacts on the mean and turbulence characteristics. A total of 23 large-eddy simulations were conducted to study these effects. The main findings of this paper include the following: 

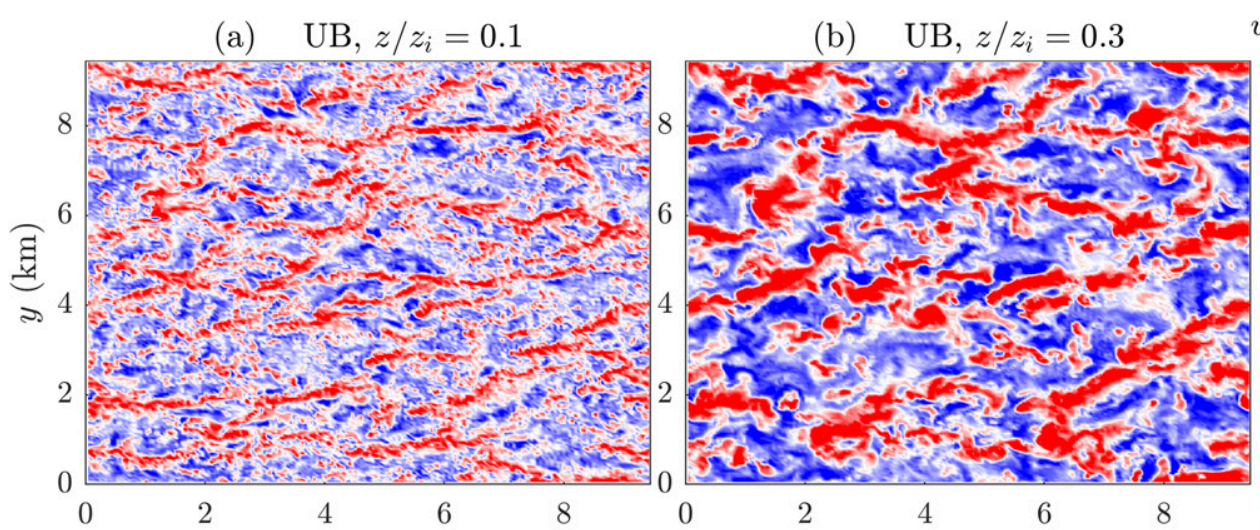

$w / G(0)$
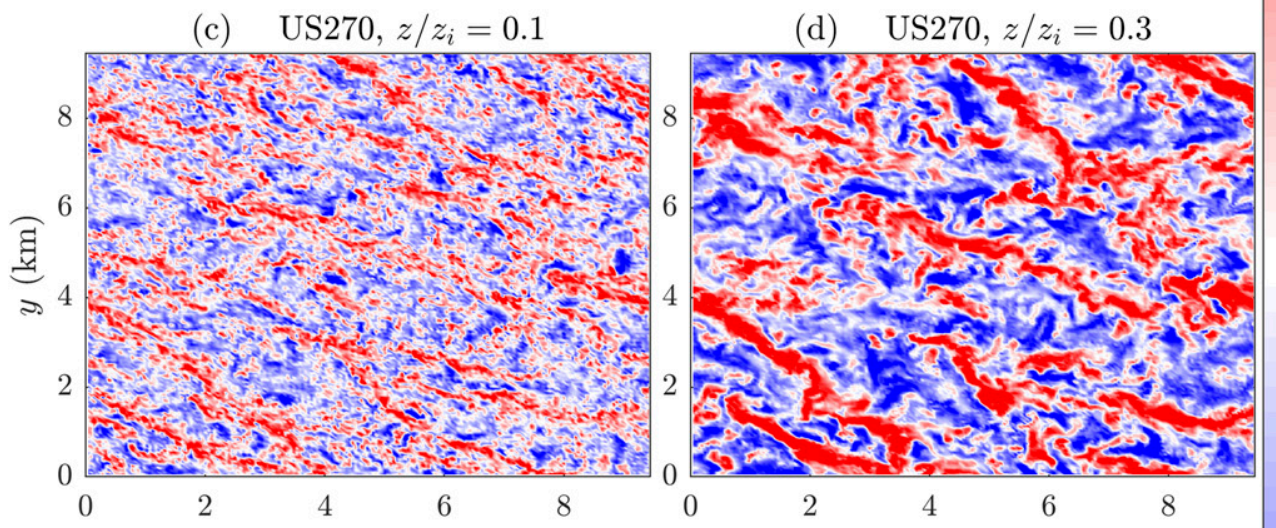

0.15

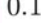

0.1

(e) US180, $z / z_{i}=0.1$

(f) $\quad \mathrm{US} 180, z / z_{i}=0.3$
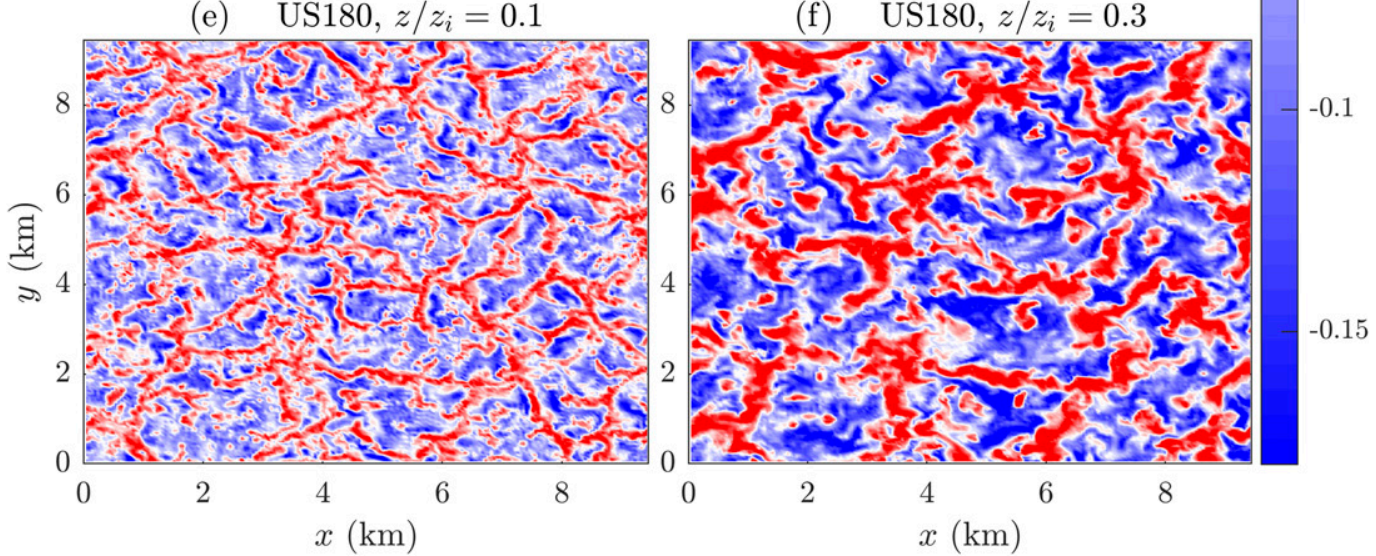

FIG. 10. The impact of the baroclinicity on the direction and formation of convective rolls and cells. (a),(c),(e) The contours of the instantaneous vertical velocity fields normalized by the geostrophic wind magnitude at the surface $G_{0}$ at $z / z_{i}=0.1$ are depicted and (b),(d),(f) the results at $z / z_{i}=0.3$.

- Baroclinicity strongly influences the Ekman turning and mean hodographs under all stabilities, even when normalized by the geostrophic wind magnitude at each elevation $G(z)$. Both weak $\left(S_{0}=0.25\right)$ and strong $\left(S_{0}=1.0\right)$ baroclinicity result in significant departures from the barotropic case and these deviations grow with increasing $S_{0}$.
- We proposed a reduced-order model to capture the effects of the baroclinicity strength and direction on the mean velocity profiles of neutral cases using a prescribed cubic eddy viscosity profile. Despite the simplicity of the model, it captures the main LES trends qualitatively well. Thus, it could be used in many applications, for example, to assess baroclinic 
effects on meteorological measurements or to estimate wind power changes due to baroclinicity.

- The velocity vectors in the ABL follow the trends of the magnitude $S_{0}$ and direction $\beta$ of the geostrophic wind vectors, particularly away from the surface layer (as turbulent stresses become less dominant in such regions). The matches between the wind velocity vectors and geostrophic wind values also depend on the stability of the ABL. They match closely in stable ABLs, where friction forces are damped as a result of stabilizing heat fluxes; on the contrary, larger departures are in unstable conditions, where friction is dominant and vertical mixing leads to more uniform wind profiles. The resulting velocity profiles are rarely observed in barotropic environments. For example, a negative geostrophic shear $\left(\beta=180^{\circ}\right)$ yields a mean velocity profile that decreases with height over more than half of the ABL depth in neutral cases, while a positive geostrophic shear $\left(\beta=0^{\circ}\right)$ strongly increases the wind speed magnitude throughout the ABL.

- The friction velocity values $u_{*}$ are strongly impacted by the baroclinicity as well. Irrespective of the static stability in diabatic simulations and baroclinicity strength in neutral cases, positive geostrophic shear and cold advection increased the friction velocity while negative geostrophic shear decreased $u_{*}$ compared to their barotropic counterparts.

- The low-level jets are highly modulated by baroclinicity. Their strength is decreased under positive geostrophic shear $\left(\beta=0^{\circ}\right)$ and intensified under negative geostrophic shear $\left(\beta=135^{\circ}\right.$ and $\left.\beta=180^{\circ}\right)$. Furthermore, positive geostrophic shear and warm advection tend to increase their height, whereas negative geostrophic shear lowers it.

- Baroclinicity generally increases shear in the outer ABL (since there always exists a vertical gradient in geostrophic wind under baroclinic conditions) and tends to make the core of the ABL more neutral by decreasing $\left|R i_{f}\right|$ away from the surface. Near the surface, these trends are reversed when there is a negative geostrophic shear.

- In some baroclinic cases, increasing TKE with height is observed, which is not seen in a comparable barotropic environment. Baroclinicity impacts the turbulence by producing shear at higher elevations. Therefore, it alters the TKE budget balance. The positive geostrophic shear increases the magnitude of the TKE budget terms and negative geostrophic shear decreases them. Baroclinicity also modifies the relative ratio of the TKE budget terms in the ABL and influences the shape of the profiles.

- The large turbulence structures are altered by baroclinicity. For instance, the positive-shear case led to a roll-type convection in the vertical velocity field while the negative-shear case led to a cellular-type convection, although the imposed heat flux and geostrophic wind values at the surface were the same in both cases. This modification is consistent with the previously reported dependence of the structural properties of barotropic ABL turbulence on $z_{i} / L$. In addition, the direction of these large structures tends to become aligned with the imposed shear in the ABL.

Our results demonstrate that baroclinicity can substantially change the characteristics of mean flow and turbulence in the ABL when compared to the classic barotropic environment, and therefore it must be accounted for in numerical/theoretical models (perhaps by using appropriate $S_{0}$ and $\beta$ ). This will result in better agreements with real-world measurements. It should also be considered when experimental observations are analyzed. Further studies are required to better understand how baroclinicity modulates other aspects such as turbulent stresses and anisotropy, turbulent transport efficiency, and conventional similarity theories in the $\mathrm{ABL}$, and to more thoroughly quantify our findings using field measurements.

Acknowledgments. The authors acknowledge support from the Princeton Environmental Institute and the National Oceanic and Atmospheric Administration (NOAA), through the Cooperative Institute for Climate Science (CICS) of Princeton University, under Grant 344-6127. Furthermore, MM and MGG acknowledge the Department of Civil Engineering and Engineering Mechanics at Columbia University for support via startup funds. The simulations were performed on the computing clusters of the National Centre for Atmospheric Research under Projects UPRI0007 and UPRI0016.

\section{APPENDIX A}

\section{Initialization and Boundary Conditions of the Simulations}

The simulations are first integrated to reach a steadystate condition on a $64 \times 64 \times 64$ numerical grid for eight inertial time scales $\tau_{\mathrm{ABL}}$. Next, all the cases are interpolated into numerical grids with $192 \times 192 \times 192$ grid nodes. The warm-up procedure is then continued for $2 \tau_{\mathrm{ABL}}$ in the high-resolution mode, to ensure development of the smallest resolved scales, equilibrium of the mean forces, and convergence of the flow statistics. Finally, statistics are computed over the last $\tau_{\mathrm{ABL}}$. Averaging over $\tau_{\mathrm{ABL}}$ (about $12.5 \mathrm{~h}$ here) is enough to fully converge 
statistics (Monin and Yaglom 1975), especially considering that we also average in the horizontal directions over the whole domain. The initial values of velocities in our code are set as the solutions to the steady neutral barotropic Ekman layer suggested by Businger (1982):

$$
\begin{aligned}
u(t & =0, z) / G=1-e^{-\gamma z} \cos (\gamma z), \\
v(t & =0, z) / G=e^{-\gamma z} \sin (\gamma z), \\
w(t=0, z) / G & =0,
\end{aligned}
$$

where, $\gamma \equiv \sqrt{f / 2 \nu_{m}}$, and $\nu_{m}=5 \mathrm{~m}^{2} \mathrm{~s}^{-1}$ is a constant eddy viscosity. Thus, upon running the code for various baroclinic cases, we found that after $\approx 8$ inertial cycles $\left(\tau_{\mathrm{ABL}}\right)$ the solutions of baroclinic cases reached a steady state for this specific initialization [Eq. (A1)]. We also checked the momentum balance of several high-resolution cases and verified this steady-state condition. We note that this long initialization is mainly done so that the computed statistics do not depend on initial conditions. This steadystate condition might not be prevalent in real-world ABLs because of many complicating factors that lead to the unsteadiness of the airflow. Nevertheless, understanding the steady-state baroclinicity effects on the spatial structures of the mean and turbulence is a necessary first step before proceeding to understanding all the complicating features of unsteady baroclinic ABLs.

In unstable and neutral simulations, the height of the domain is $L_{z}=1500 \mathrm{~m}$ with a horizontal size of $L_{x}=$ $L_{y}=2 \pi L_{z}$. In stable simulations, we set $L_{z}=1100 \mathrm{~m}$ to obtain a higher resolution. Boundary conditions are periodic in the horizontal directions. For the bottom boundary, a local equilibrium logarithmic-wall model based on spatially filtered velocities is used, to evaluate surface stresses (Bou-Zeid et al. 2005), and a constant surface heat flux is imposed for the scalar equation. The aerodynamic roughness length $z_{0}$ is set to $0.1 \mathrm{~m}$ for all the considered cases. The surface value of the geostrophic wind at the wall $G_{0}$ is always equal to the barotropic geostrophic value $\left(8 \mathrm{~m} \mathrm{~s}^{-1}\right.$ here). Under neutral conditions, normalization by $G_{0}$ makes its value irrelevant; however, this dimensional value is important in diabatic simulations (relative to the surface heat flux) and affects stability parameters like Obukhov length and Richardson number.

For the top boundary, we use a stress-free and impermeable $(w=0)$ boundary condition for the velocity field and an inversion layer with $0.03 \mathrm{~K} \mathrm{~m}^{-1}$ slope is imposed above $z_{i}=800 \mathrm{~m}$ in the stable cases and $1000 \mathrm{~m}$ in the neutral and unstable runs. The inversion layer imposes a limit on the height and growth of the ABL. We note that in this study we did not aim to investigate the dynamical evolution of the ABL height, since we focus on the steady-state baroclinic ABLs under various imposed surface heat flux conditions. In stable cases, we also impose a sponge layer with a Rayleigh damping method (Israeli and Orszag 1981) at $z=825 \mathrm{~m}$ to damp all perturbations thus preventing the reflection of internal waves.

\section{APPENDIX B}

\section{Comparison of the Results of our Baroclinic LES Setup with Other Codes}

We compared our LES results with two neutral cases of Brown (1996). Brown (1996) used a nonuniform vertical grid with a resolution of $40 \times 40 \times 32$ while we use uniform grids with a resolution of $128 \times 128 \times 128$. His boundary conditions were periodic in the horizontal directions, with a rough wall model at the bottom, and a rigid lid with zero stress and heat flux at the top. Brown (1996) used the static Smagorinsky model (imposed nondynamic coefficient) for parameterizing the SGS components and hence we also use the static Smagorisnky model in these two simulations to allow direct comparison. The results shown in the supplemental material indicate a good agreement between our LESs and his numerical results. We note that in other simulations in the paper, we use a scale-dependent Lagrangian dynamic Smagorinsky SGS model (BouZeid et al. 2005). This model reproduces the logarithmic region in high-Reynolds-number simulations, which do not resolve the viscous sublayer, better than other SGS models like the scale-invariant dynamic Smagorinsky models. Furthermore, the velocity profiles of this model match better with an experimental data compared to other SGS models [see Bou-Zeid et al. (2005) for SGS models comparison].

Note that Sorbjan (2004) only shows his results at $t / \tau_{\mathrm{ABL}} \approx 0.25$; however, we believe that in order to reach equilibrium and overcome the initial-condition effects, the simulations have to be integrated at least about 2 times the inertial time scale $\left(2 \tau_{\mathrm{ABL}}\right)$. We concur with Sorbjan's (2004) results for earlier times in our simulations, but we do not show them here since they have not reached the full equilibrium with the imposed geostrophic forcing.

Brown (1996) indicated that the time series of $u_{*}$ were close to achieving equilibrium after $t / \tau_{\mathrm{ABL}} \approx 1.6$ and so he ran the simulations up to this time and we compare our results with his cases here. These comparisons demonstrate that our baroclinic setup matches the results of other available codes well, which serves as a check on the simulation setup and validity. 


\section{REFERENCES}

Albertson, J. D., and M. B. Parlange, 1999: Natural integration of scalar fluxes from complex terrain. Adv. Water Resour., 23, 239-252, https://doi.org/10.1016/S0309-1708(99)00011-1.

Arya, S. P. S., and J. C. Wyngaard, 1975: Effect of baroclinicity on wind profiles and the geostrophic drag law for the convective planetary boundary layer. J. Atmos. Sci., 32, 767-778, https://doi.org/ 10.1175/1520-0469(1975)032<0767:EOBOWP>2.0.CO;2.

Basu, S., J. Vinuesa, and A. Swift, 2008: Dynamic LES modeling of a diurnal cycle. J. Appl. Meteor. Climatol., 47, 1156-1174, https://doi.org/10.1175/2007JAMC1677.1.

Berger, B. W., and B. Grisogono, 1998: The baroclinic, variable eddy viscosity Ekman layer: An approximate analytical solution. Bound.-Layer Meteor., 87, 363-380, https://doi.org/10.1023/A: 1001076030166.

Blackadar, A., 1957: Boundary layer wind maxima and their significance for the growth of nocturnal inversions. Bull. Amer. Meteor. Soc., 38, 283-290, https://doi.org/10.1175/1520-0477-38.5.283.

Bou-Zeid, E., C. Meneveau, and M. Parlange, 2005: A scaledependent Lagrangian dynamic model for large eddy simulation of complex turbulent flows. Phys. Fluids, 17, 025105, https://doi.org/10.1063/1.1839152.

— J. Overney, B. D. Rogers, and M. B. Parlange, 2009: The effects of building representation and clustering in large-eddy simulations of flows in urban canopies. Bound.-Layer Meteor., 132, 415-436, https://doi.org/10.1007/s10546-009-9410-6.

Brown, A. R., 1996: Large-eddy simulation and parametrization of the baroclinic boundary-layer. Quart. J. Roy. Meteor. Soc., 122, 1779-1798, https://doi.org/10.1002/qj.49712253603.

— A. C. M. Beljaars, and H. Hersbach, 2006: Errors in parametrizations of convective boundary-layer turbulent momentum mixing. Quart. J. Roy. Meteor. Soc., 132, 1859-1876, https://doi.org/10.1256/qj.05.182.

Businger, J. A., 1982: Equations and concepts. Atmospheric Turbulence and Air Pollution Modelling, F. T. M. Nieuwstadt and H. van Dop, Eds., D. Reidel Publishing Company, 1-36, https://doi.org/10.1007/978-94-010-9112-1.

Cantero, M. I., S. Balachandar, M. H. García, and D. Bock, 2008: Turbulent structures in planar gravity currents and their influence on the flow dynamics. J. Geophys. Res., 113, 1-22, https://doi.org/10.1029/2007JC004645.

Chow, F. K., and R. L. Street, 2009: Evaluation of turbulence closure models for large-eddy simulation over complex terrain: Flow over Askervein Hill. J. Appl. Meteor. Climatol., 48, 1050-1065, https://doi.org/10.1175/2008JAMC1862.1.

Deardorff, J. W., 1970: Convective velocity and temperature scales for the unstable planetary boundary layer and for Rayleigh convection. J. Atmos. Sci., 27, 1211-1213, https://doi.org/ 10.1175/1520-0469(1970)027<1211:CVATSF>2.0.CO;2.

-, 1972: Numerical investigation of neutral and unstable planetary boundary layers. J. Atmos. Sci., 29, 91-115, https://doi.org/ 10.1175/1520-0469(1972)029<0091:NIONAU>2.0.CO;2.

Djolov, G. D., D. L. Yordanov, and D. E. Syrakov, 2004: Baroclinic planetary boundary-layer model for neutral and stable stratification conditions. Bound.-Layer Meteor., 111, 467-490, https://doi.org/10.1023/B:BOUN.0000016507.94623.e5.

Ekman, V. W., 1905: On the influence of the Earth's rotation on ocean-currents. Ark. Mat. Astron. Fys., 2, 1-52.

Floors, R., A. Pena, and S.-E. Gryning, 2015: The effect of baroclinicity on the wind in the planetary boundary layer. Quart. J. Roy. Meteor. Soc., 141, 619-630, https://doi.org/ 10.1002/qj.2386.
Gayen, B., S. Sarkar, and J. R. Taylor, 2010: Large eddy simulation of a stratified boundary layer under an oscillatory current. J. Fluid Mech., 643, 233-266, https://doi.org/10.1017/ S002211200999200X.

Grisogono, B., 1995: A generalized Ekman layer profile with gradually varying eddy diffusivities. Quart. J. Roy. Meteor. Soc., 121, 445-453, https://doi.org/10.1002/qj.49712152211.

Gula, J., M. J. Molemaker, and J. C. McWilliams, 2014: Submesoscale cold filaments in the Gulf Stream. J. Phys. Oceanogr., 44, 2617-2643, https://doi.org/10.1175/JPO-D-14-0029.1.

Hess, G. D., 1973: On Rossby-number similarity theory for a baroclinic planetary boundary layer. J. Atmos. Sci., 30, 1722-1723, https:// doi.org/10.1175/1520-0469(1973)030<1722:ORNSTF $>2.0$.CO;2.

- 2004: The planetary boundary layer, capped by an inversion: Extension to baroclinic and stable conditions. Aust. Meteor. Mag., 53, 95-101.

Holtslag, A. A. M., and Coauthors, 2013: Stable atmospheric boundary layers and diurnal cycles: Challenges for weather and climate models. Bull. Amer. Meteor. Soc., 94, 1691-1706, https://doi.org/10.1175/BAMS-D-11-00187.1.

Hoxit, L., 1974: Planetary boundary layer winds in baroclinic conditions. J. Atmos. Sci., 31, 1003-1020, https://doi.org/10.1175/ 1520-0469(1974)031<1003:PBLWIB > 2.0.CO;2.

Huang, J., and E. Bou-Zeid, 2013: Turbulence and vertical fluxes in the stable atmospheric boundary layer. Part I: A large-eddy simulation study. J. Atmos. Sci., 70, 1513-1527, https://doi.org/ 10.1175/JAS-D-12-0167.1.

Israeli, M., and S. A. Orszag, 1981: Approximation of radiation boundary conditions. J. Comput. Phys., 41, 115-135, https:// doi.org/10.1016/0021-9991(81)90082-6.

Knaepen, B., O. Debliquy, and D. Carati, 2002: Subgrid-scale energy and pseudo pressure in large-eddy simulation. Phys. Fluids, 14, 4235-4241, https://doi.org/10.1063/1.1514643.

Kumar, V., G. Svensson, M. Holtslag, C. Meneveau, and M. B. Parlange, 2010: Impact of surface flux formulations and geostrophic forcing on large-eddy simulations of diurnal atmospheric boundary layer flow. J. Appl. Meteor. Climatol., 49, 1496-1516, https://doi.org/10.1175/2010JAMC2145.1.

Kundu, P. K., and I. M. Cohen, 2008: Fluid Mechanics. Academic Press, $872 \mathrm{pp}$.

Lilly, D. K., 1966: On the instability of Ekman boundary layer. J. Atmos. Sci., 23, 481-494, https://doi.org/10.1175/1520-0469 (1966) $023<0481$ :OTIOEB $>2.0$. CO;2.

Mahrt, L. J., and W. Schwerdtfeger, 1970: Ekman spirals for exponential thermal wind. Bound.-Layer Meteor., 1, 137-145, https://doi.org/10.1007/BF00185735.

Momen, M., and E. Bou-Zeid, 2015: Analytical damped-oscillator models for unsteady atmospheric boundary layers. 68th Annual Meeting of the American Physical Society Division of Fluid Dynamics, Vol. 60, Boston, MA, American Physical Society, Abstract: BAPS.2015.DFD.KP1.148, http://meetings. aps.org/Meeting/DFD15/Event/254408.

— models of the unsteady Ekman boundary layer. J. Atmos. Sci., 73, 25-40, https://doi.org/10.1175/JAS-D-15-0038.1.

— mospheric flows. 69th Annual Meeting of the American Physical Society Division of Fluid Dynamics, Vol. 61, Portland, OR, American Physical Society, Abstract: M13.004, http://apsdfd2016pdx.org/.

_ and - 2017a: Mean and turbulence dynamics in unsteady Ekman boundary layers. J. Fluid Mech., 816, 209-242, https:// doi.org/10.1017/jfm.2017.76. 
and $-2017 \mathrm{~b}$ : Analytical reduced models for non-stationary diabatic atmospheric boundary layers. Bound.-Layer Meteor., 164, 383-399, https://doi.org/10.1007/s10546-017-0247-0.

— J. D. Wood, K. A. Novick, R. Pangle, W. T. Pockman, N. G. McDowell, and A. G. Konings, 2017a: Interacting effects of leaf water potential and biomass on vegetation optical depth. J. Geophys. Res. Biogeosci., 122, 3031-3046, https://doi.org/ 10.1002/2017JG004145.

— Z. Zheng, E. Bou-Zeid, and H. A. Stone, 2017b: Inertial gravity currents produced by fluid drainage from an edge. J. Fluid Mech., 827, 640-663, https://doi.org/10.1017/jfm.2017.480.

Monin, A. S., and A. M. Obukhov, 1954: Basic laws of turbulent mixing in the surface layer of the atmosphere. Tr. Geofiz. Inst., Akad. Nauk SSSR, 24, 163-187.

__ and A. M. Yaglom, 1975: Statistical Fluid Mechanics. MIT Press, 769 pp.

Nieuwstadt, F. T. M., 1983: On the solution of the stationary, baroclinic Ekman-layer equations with a finite boundary-layer height. Bound.-Layer Meteor., 26, 377-390, https://doi.org/ 10.1007/BF00119534.

Nunalee, C. G., and S. Basu, 2014: Mesoscale modeling of coastal lowlevel jets: Implications for offshore wind resource estimation Wind Energy, 17, 1199-1216. https://doi.org/10.1002/we.1628.

O'Brien, J. J., 1970: A note on the vertical structure of the eddy exchange coefficient in the planetary boundary layer. J. Atmos. Sci., 27, 1213-1215, https://doi.org/10.1175/15200469(1970)027<1213:ANOTVS > 2.0.CO;2.

Pedersen, J. G., M. Kelly, S. E. Gryning, and B. Brümmer, 2013: The effect of unsteady and baroclinic forcing on predicted wind profiles in large eddy simulations: Two case studies of the daytime atmospheric boundary layer. Meteor. Z., 22, 661-674, https://doi.org/10.1127/0941-2948/2013/0477.

_ , S. E. Gryning, and M. Kelly, 2014: On the structure and adjustment of inversion-capped neutral atmospheric boundarylayer flows: Large-eddy simulation study. Bound.-Layer Meteor., 153, 43-62, https://doi.org/10.1007/s10546-014-9937-z.

Ramos da Silva, R., and R. Avissar, 2006: The hydrometeorology of a deforested region of the Amazon basin. J. Hydrometeor., 7, 1028-1042, https://doi.org/10.1175/JHM537.1.

Rizza, U., M. M. Miglietta, O. C. Acevedo, V. Anabor, G. A. Degrazia, A. G. Goulart, and H. R. Zimmerman, 2013: Largeeddy simulation of the planetary boundary layer under baroclinic conditions during daytime and sunset turbulence. Meteor. Appl., 20, 56-71, https://doi.org/10.1002/met.1284.

Salesky, S. T., and M. Chamecki, 2012: A similarity model of subfilter-scale energy for large-eddy simulations of the atmospheric boundary layer. Bound.-Layer Meteor., 145, 69-91, https://doi.org/10.1007/s10546-011-9618-0.
— - - , and E. Bou-Zeid, 2017: On the nature of the transition between roll and cellular organization in the convective boundary layer. Bound.-Layer Meteor., 163, 41-68, https://doi.org/10.1007/s10546-016-0220-3.

Shah, S., and E. Bou-Zeid, 2014: Very-large-scale motions in the atmospheric boundary layer educed by snapshot proper orthogonal decomposition. Bound.-Layer Meteor., 153, 355-387, https://doi.org/10.1007/s10546-014-9950-2.

Sheppard, P. A., H. Charnock, and J. R. D. Francis, 1952: Observations of the westerlies over the sea. Quart. J. Roy. Meteor. Soc., 78, 563-582, https://doi.org/10.1002/qj.49707833807.

Sorbjan, Z., 2004: Large-eddy simulations of the baroclinic mixed layer. Bound.-Layer Meteor., 112, 57-80, https://doi.org/10.1023/ B:BOUN.0000020161.99887.b3.

Stull, R., 1988: An Introduction to Boundary Layer Meteorology. 1st ed. Kluwer Academic Publishers, 378 pp.

Sun, J., D. H. Lenschow, L. Mahrt, and C. Nappo, 2013: The relationships among wind, horizontal pressure gradient, and turbulent momentum transport during CASES-99. J. Atmos. Sci., 70, 3397-3414, https://doi.org/10.1175/JAS-D-12-0233.1.

Tan, Z. M., 2001: An approximate analytical solution for the baroclinic and variable eddy diffusivity semi-geostrophic Ekman boundary layer. Bound.-Layer Meteor., 98, 361-385, https:// doi.org/10.1023/A:1018708726112.

Tennekes, H., and J. Lumley, 1972: A First Course in Turbulence. MIT Press, $320 \mathrm{pp}$.

Vallis, G. K., 2006: Atmospheric and Oceanic Fluid Dynamics: Fundamentals and Large-Scale Circulation. 1st ed. Cambridge University Press, $745 \mathrm{pp}$.

Van de Wiel, B. J. H., F. Moene, G. J. Steeneveld, P. Baas, F. C. Bosveld, and M. Holtslag, 2010: A conceptual view on inertial oscillations and nocturnal low-level jets. J. Atmos. Sci., 67, 2679-2689, https://doi.org/10.1175/2010JAS3289.1.

Venkatesh, S., and G. T. Csanady, 1974: A baroclinic planetary boundary-layer model, and its application to the Wangara data. Bound.-Layer Meteor., 5, 459-473, https://doi.org/10.1007/ BF00123492.

Wyngaard, J. C., 2010: Turbulence in the Atmosphere. Cambridge University Press, 393 pp.

Young, G. S., D. A. R. Kristovich, M. R. Hjelmfelt, and R. C. Foster, 2002: Rolls, streets, waves, and more: A review of quasitwo-dimensional structures in the atmospheric boundary layer. Bull. Amer. Meteor. Soc., 83, 997-1002, https://doi.org/10.1175/ 1520-0477(2002)083<0997:RSWAMA > 2.3.CO;2

Zilitinkevich, S., and I. N. Esau, 2003: The effect of baroclinicity on the equilibrium depth of neutral and stable planetary boundary layers. Quart. J. Roy. Meteor. Soc., 129, 3339-3356, https:// doi.org/10.1256/qj.02.94. 